\title{
AN INDUSTRIAL GAUGE FOR MEASURING THE PHASE DISTRIBUTION OF GALVANNEAL
}

\section{FINAL REPORT}

$11 / 30 / 92-11 / 22 / 95$

\author{
By \\ Christopher Burnett \\ Roland Gouel \\ James R. Phillips
}

January 19, 1996

Work Performed Under Cooperative Agreement No. DE-FC07-93ID13205

Prepared for

American Iron and Steel Institute

Advanced Process Control Program

\author{
Prepared by \\ Measurex-DMC \\ 15884 Gaither Drive \\ Gaithersburg, MD 20877 \\ (301) $948-2450$
}




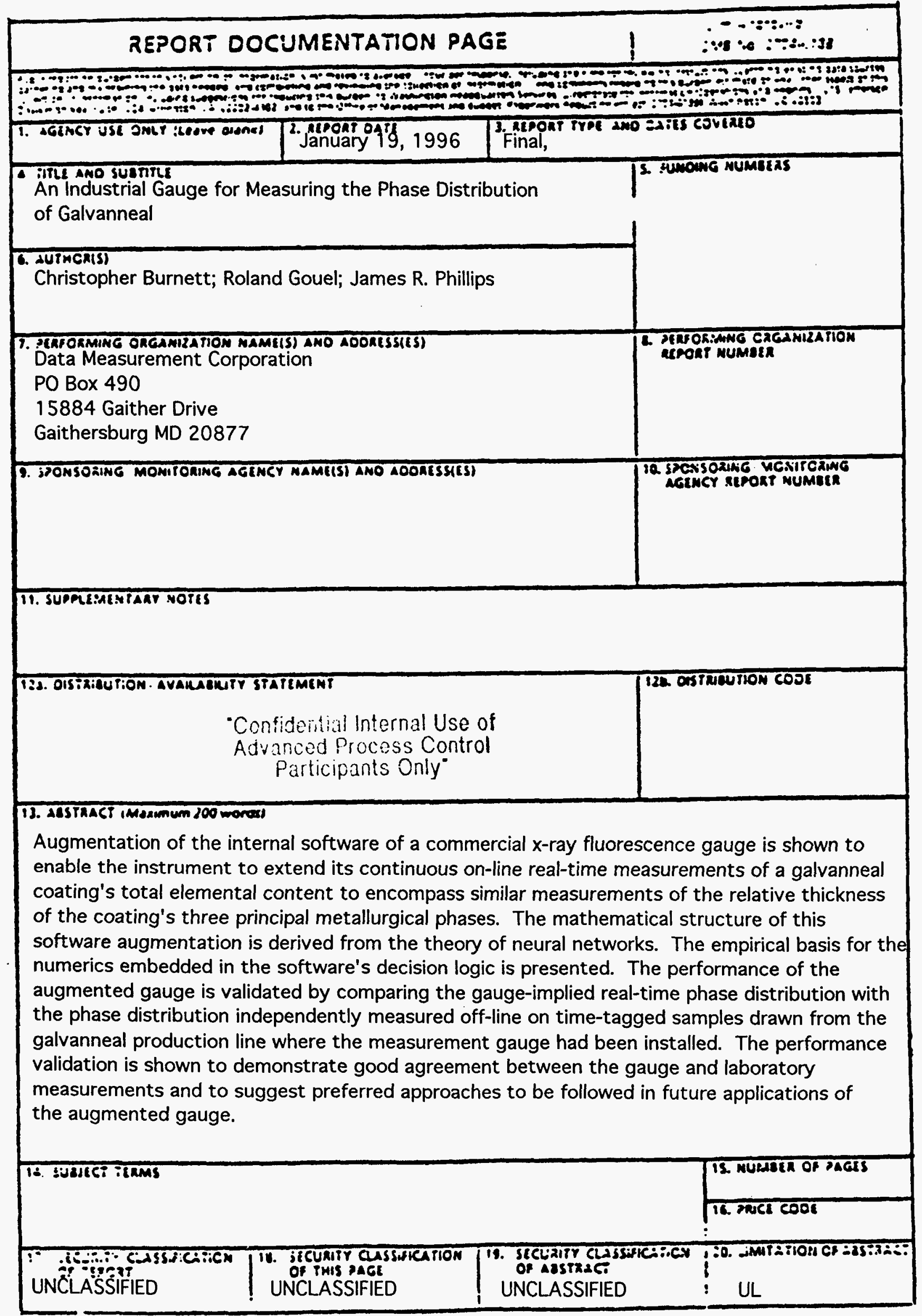




\section{FOREWORD}

The authors view this report as a tribute to Dominique Gignoux, Data Measurement Corporation's Founder and President, whose vision and enthusiasm animated the project summarized herein. His friends and associates mourn his recent death. That tragic event's timing, two weeks before publication of this document, has denied it his final approbation and deprived the authors of his guidance and inspiration.

The authors gratefully acknowledge the valuable support, suggestions, and technical stimulation from

- the project's sponsors at the U.S. Department of Energy and the American Iron and Steel Institute,

- the project's other principal participants at Inland Steel Company, Jet Propulsion Laboratory, and Stelco, Inc.,

- other contributors at Bethlehem Steel, Dofasco, LTV Steel, and Weirton Steel. 
Page

Foreword

Table of Contents

ii

List of Figures

List of Tables

EXECUTIVE SUMMARY

iii

iv

v

vi

1. INTRODUCTION 1

2. GAUGE DEVELOPMENT 3

2.1 Creating Galvanneal Sample and Training Sets 7

2.2 Hardware Base: DMC800 Zinc/Iron Coating Gauge 10

2.3 Software Augmentation 15

2.4 Validation of the Augmented Gauge 27

3. CONCLUSIONS AND RECOMMENDATIONS 46

REFERENCES 48

Appendix I: JPL's Final Report for Project E: March 1, 1995

Appendix II: Description of the DMC800 Zinc/Iron Coating Gauge 


\section{LIST OF FIGURES}

Figure

Page

1 Traditional Phases of Galvanneal 5

2 Iron/Zinc Alloy Phases 6

3 Pilot Coating Line Test Setup $\quad 8$

4 Ideal Sample Map - Graph 1 11

5 Sample Map - Graph 2 12

6 X-Ray Fluorescence - Orthogonal Incidence 13

7 X-Ray Fluorescence - Shallow Incidence 14

8 Typical Zinc/Iron System Block Diagram 16

9 First Model - Laboratory Accuracy \% Zeta 28

10 First Model - Laboratory Accuracy \% Delta 29

11 First Model - Laboratory Accuracy \% Gamma 30

12 On-Line Testing Block Diagram 31

13 Static Analysis of On-line Model 2\% Zeta 32

14 Static Analysis of On-line Model 2\% Delta 33

15 Static Analysis of On-line Model 2\% Gamma 34

16 Voltage Map of Sample Data - Stelco and Inland 35

17 Stelco Data Model Accuracy \% Zeta 36

18 Stelco Data Model Accuracy \% Delta 37

19 Stelco Data Model Accuracy \% Gamma 38

20 Combined Data Model Accuracy \% Zeta 43

21 Combined Data Model Accuracy \% Delta 44

22 Combined Data Model Accuracy \% Gamma 45 


\section{LIST OF TABLES}

Table

Page

$1 \quad$ Training-Set for Model 1

2 Neural Network Coupling- and Bias-Coefficient Arrays for 22 Model 1

3 Measured vs. Computed Phases for Model 1

4 Training-Set for Model 2

5 Neural Network Coupling- and Bias-Coefficient Arrays for 25 Model 2

6 Measured vs. Computed Phases for Model 2

7 Training-Set for Composite Model 40

8 Neural Network Coupling- and Bias-Coefficient Arrays for the 41 Composite Model

9 Measured vs. Computed Phases for the Composite Model 


\section{EXECUTIVE SUMMARY}

This report summarizes the technical contributions of Measurex-DMC (hereinafter referred to as Data Measurement Corporation) to Project E ("Phase Distribution of Galvanneal") of the "Advanced Process Control Research Program for the Steel Industry," a cost-shared program sponsored by the U.S. Department of Energy (DOE) and the American Iron and Steel Institute (AISI). Galvanneal is a relatively new process wherein steel is coated with a mixture of zinc and iron and yields steel sheet with superior weldability and paintability characteristics. It has been recognized empirically that the coating's spatial distribution of certain metallurgical phases of zinc and iron dictates the preferred galvanneal properties. Accordingly, the objective of this project was the development of an on-line instrument that employs $x$-ray fluorescence and software analytical techniques for continuous, rapid, non-destructive, and accurate measurement of the galvanneal phase distribution (i.e., the relative thickness of the coating's three principal metallurgical phases). Such an instrument is an indispensable ingredient of an on-line feedback system for controlling the properties of galvanneal steel.

This project's team consisted of Inland Steel Company and Stelco, Inc. as the primary steel companies, the Jet Propulsion Laboratory (JPL) of Caltech, and Data Measurement Corporation (DMC). Bethlehem Steel, Dofasco, LTV Steel, and Weirton Steel also made valuable technical contributions to the project. By systematically varying operating conditions, substrate thickness, and coating mass, Inland Steel produced at its pilot coating facility over a hundred galvanneal samples, whose properties encompassed all the combinations deemed commercially valuable. JPL conducted laboratory measurements of the phase distribution of the Inland-produced samples. JPL's measurement process consisted of direct mechanical scale-alignment with an optical photomicrograph (magnified a thousand fold) of a sample cross-section after the sample had been etched to delineate and visualize the boundaries of the galvanneal phases. DMC non-destructively measured the elemental composition and coating weight of the Inland-produced samples. These measurements were made by DMC in a laboratory version of its commercially available DMC800 Zinc/Iron Coating Gauge; the laboratory and on-line versions use identical measurement hardware and sense the $\mathrm{x}$-ray fluorescence identically. 
DMC developed an original mathematical representation in which two internal signals (i.e., " the relative zinc and iron voltages") routinely generated in the measuring head of the DMC800 Zinc/Iron Coating Gauge provide the basis for estimating the galvanneal phase distribution. (For each Inland-produced sample measured in the laboratory version of this gauge, the values of these two internally generated signals had been recorded.) The structure of DMC's mathematical representation is drawn from the theory of neural networks. Within this project, any specific numerical embodiment of the underlying mathematical representation was called a "math model." All such math models share the same generic conceptual framework and differ only in the numerical values assigned to their constituent parameters. As with any pattern recognition scheme, a "training-set" is needed in order to flesh out the numerics of the representation's decision logic. All the training-sets considered during this phase of the project originated as subsets of the total population of Inland-produced samples. Program reviews produced a consensus that any candidate training-set should discard every sample that contained a coating phase consisting of pure zinc. Two training-sets meeting this criterion were created, containing seventyfour and sixty-six samples, respectively; the smaller set reflects discarding eight additional questionable samples. For each training-set, the mathematical representation's input and output parameters for each sample consisted of the two DMC-measured signals described above and the three JPL-measured relative phases, respectively. The sole additional input property assigns each sample to one of the three mutually exclusive classes (viz., extra-low-carbon, interstitialfree, and rephosphorized) of galvanneal substrate; this property is both fundamental and static, inasmuch as it doesn't change during a production run. The resulting numerical incarnations of DMC's mathematical representation were called Math Models 1 and 2 according as the larger or smaller, respectively, of the two training-sets had served to characterize the model's numerics completely.

The data for a completely independent test and evaluation of the DMC approach were obtained at the Stelco Hilton Works' Z-Line, where a DMC800 Zinc/Iron Coating Gauge was already installed and operational. In three days of on-line testing, the two gauge-measured signals (i.e., the relative zinc and iron voltages) for each of the galvanneal's top and bottom surfaces were recorded online, along with the corresponding phase distributions estimated by Math Models 1 and 2 running on a $\mathrm{PC}$ installed by $\mathrm{DMC}$ for this test sequence. In addition, 
some twenty-six samples from the Stelco coils produced during these three days were selected for off-line examination. For these twenty-six samples, DMC made and recorded the same measurements in the same laboratory version of the online gauge as used for the Inland-produced samples. For these twenty-six samples, Stelco made the phase distribution measurements by using its own prescribed metallographic techniques, which differed from JPL's. Application of the unmodified Math Models 1 and 2 to these twenty-six samples yielded phase distribution estimates in substantial disagreement with the Stelco-measured values. This result was not unanticipated, in view of recognized manufacturer-tomanufacturer differences in galvanneal production and metallography techniques from one steelmaker to another.

An extension of the DMC approach showed that such disparities could be circumvented. The specific extension was predicated on an enlarged training-set consisting of the previous Math Model 2 (Inland- produced and JPLmetallographed) samples plus the newer twenty-six (Stelco-produced and Stelcometallographed) samples; moreover, an additional input property identified the sample as having been produced by Inland or Stelco. The resulting numerical embodiment of DMC's mathematical representation is called the Composite Math Model and provides phase distribution estimates which agree well with the independently measured phase distribution for all samples for which such measurements are available. These results serve not only to validate DMC's basic approach, but also to suggest precautions to be observed in porting the augmented DMC gauge from one setting to another.

In brief, the joint efforts of the project's participants have achieved Project E's objective : the development of an on-line instrument for continuous reading of the zinc-iron phase distribution in the galvanneal coating, using $x$-ray fluorescence and software analytical techniques. 


\section{INTRODUCTION}

No American industry is more fundamental or competitive - both domestically and internationally - than the steel industry. Global challenges have intensified the American steel industry's historical commitment to improving the quality, energy-cost, yield, and efficiency of its production. The industry's technical management has recognized that current research developments provide both the "technology push and pull" (i.e., potential reward and nascent feasibility) to achieve such product improvements via on-line, realtime, continuous feedback control of the steelmaking process. By early 1992, the industry's collaborative research had defined the Advanced Process Control Program, containing six technology projects each aimed at a specific contribution to the overall process-control strategy. In its role as prime contractor for the entire program, the American Iron and Steel Institute (AISI) proposed the program to the Office of Industrial Processes of the US Department of Energy (DOE) for funding under the Steel and Aluminum Energy Conservation and Technology Competitiveness Act of 1988. The proposal embodied cost-sharing between DOE and AISI and thereby reflects the National Energy Strategy's suggestion that "...funding be increased for cost-shared research projects which improve energy efficiency and productivity in major energy-using industries."

Two major ingredients of a generic control system are its sensor and actuator subsystems. Each of the constituent projects within the Advanced Process Control Program was directed toward a distinctive sensor or actuator technology, and each project contributed predominantly to one of the steel industry's major processes, viz.,

- primary steelmaking,

- continuous casting,

- rolling and annealing,

- coated products.

Participants in the Advanced Process Control Program included universities, national laboratories, advanced technology companies, and the steel industry. 
Within each of the program's six projects, each participant assumed one (or more) of the following roles:

- research partner,

- commercialization partner,

- sponsoring steel company.

This management approach via partnering was intended to broaden participants' perspective, highlight interface issues early, and accelerate the development cycle for the project's technology.

This report summarizes the technical contributions of Data Measurement Corporation (DMC) to one of the Advanced Process Control Program's six projects: Project E. This project was aimed at developing a sensor for measuring certain properties of a specific family of coated products. Section 2 below provides an overview of the project, summarizes the contributions from DMC's project partners, presents the technical approach for augmenting the internal software of a commercial DMC gauge, and demonstrates that the augmented gauge met Project E's objective. Section 3 below presents conclusions and recommendations emerging from this effort. This report also includes Appendices which present additional technical detail and which make the report more nearly self-contained. 


\section{GAUGE DEVELOPMENT}

The team for Project E ("Phase Distribution of Galvanneal") of the Advanced Process Control Research Program for the Steel Industry consisted originally of Inland Steel Company as the sponsoring steel company, the Jet Propulsion Laboratory (JPL) of Caltech as a research partner, and Data Measurement Corporation (DMC) as both a research partner and the commercialization partner. The motivation for this project was stated succinctly in the program proposal (Ref. 1, p. 9):

"Galvannealing is the process of coating steel with a mixture of zinc and iron. Sheet product with this coating exhibits superior weldability and paintability characteristics and is replacing galvanized steel in many product applications including automobile body panels. ...In the galvanneal process, the strip passes first through a zinc bath (as with conventional galvanizing) and then through a galvannealing furnace which extends the time the steel is in contact with the molten zinc, enhancing the migration of iron from the steel into the zinc. The iron and zinc do not mix randomly and there are preferred phases of iron and zinc which yield the most desirable properties. The zinc-iron phase distribution is a function of the time-temperature heating cycle in the galvanneal furnace, and dictates galvanneal properties. ...At present, the galvanneal process is controlled by a basic trial and error approach as on-line temperature measurements fail because conventional optical pyrometry requires the knowledge of emissivity, a parameter that is changing during the galvanneal process. ... The critical parameter affected by temperature is the phase distribution of the zinc-iron coating which, as noted above, dictates properties. This can presently only be measured off-line in the laboratory. Offline testing of phase distribution has the same quality and productivity penalties as were described ...previous(ly)." 
The project's objective was stated even more succinctly (Ref. 1, p. 10, emphasis added):

\section{"The 'Phase Measurement of Galvanneal' project is directed at the development of an on-line instrument for continuous reading of the zinc-iron phase distribution in the coating, using $x$-ray fluorescence and software analytical techniques."}

The galvanneal phase distribution is the relative thickness of the coating's three principal metallurgical phases, viz., the gamma, delta, and zeta phases, containing compounds such as $\mathrm{Fe} \mathrm{Zn}_{3}, \mathrm{Fe} \mathrm{Zn}_{7}$, and Fe $\mathrm{Zn}_{13}$, respectively, and hence exhibiting iron concentrations of 22 to $24 \%, 9$ to $18 \%$, and 5 to $7 \%$, respectively. (A fourth phase - the eta phase - consists of pure zinc; the presence of such a layer in galvanneal is generally undesirable commercially.) Figure 1 illustrates the generic phases of galvanneal and Figure 2 illustrates one desirable and two undesirable realizations of galvanneal phases. To interpret these illustrations, it should be noted that ten micrometers is, to within a factor of two, a typical value for the total thickness of the galvanneal coating.

Project E's proposed technical approach consisted of sequentially

- producing galvanneal samples with physical properties realistic and varied enough to span the entire commercially valuable range of the relevant parameter space,

- making off-line laboratory measurements of the phase distributions of every such sample,

- measuring the signals generated in an existing DMC x-ray fluorescence gauge by every such sample,

- employing the measurements from the preceding two steps as a training set for developing a mathematical representation whereby the gaugegenerated signals provide the basis for estimating the galvanneal phase distribution, 


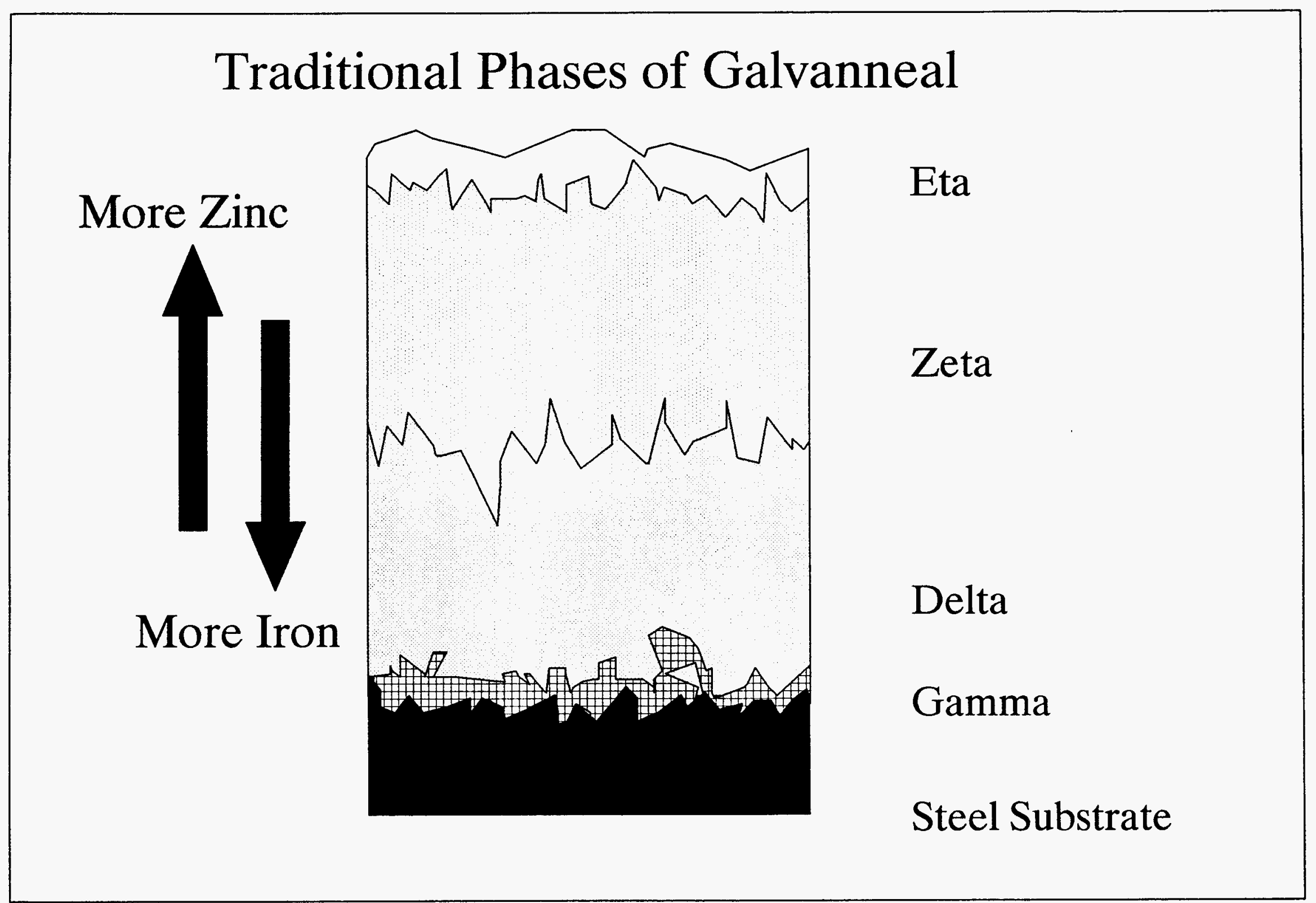




\section{Phase Measurement of Galvanneal}

\section{IRON/ZINC ALLOY PHASES}

\section{Underalloyed Structure:}

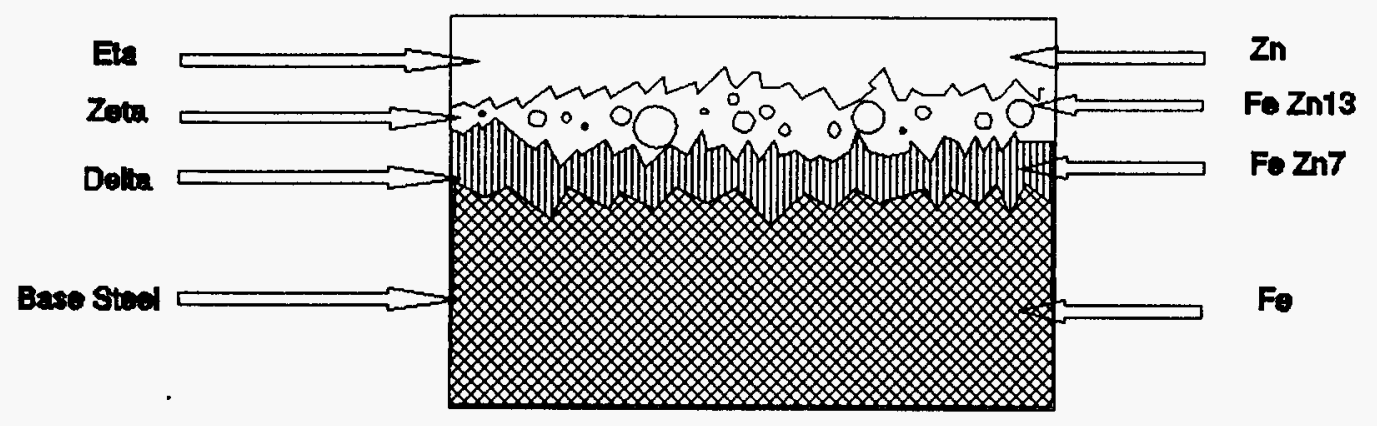

\section{Overalloyed Structure:}

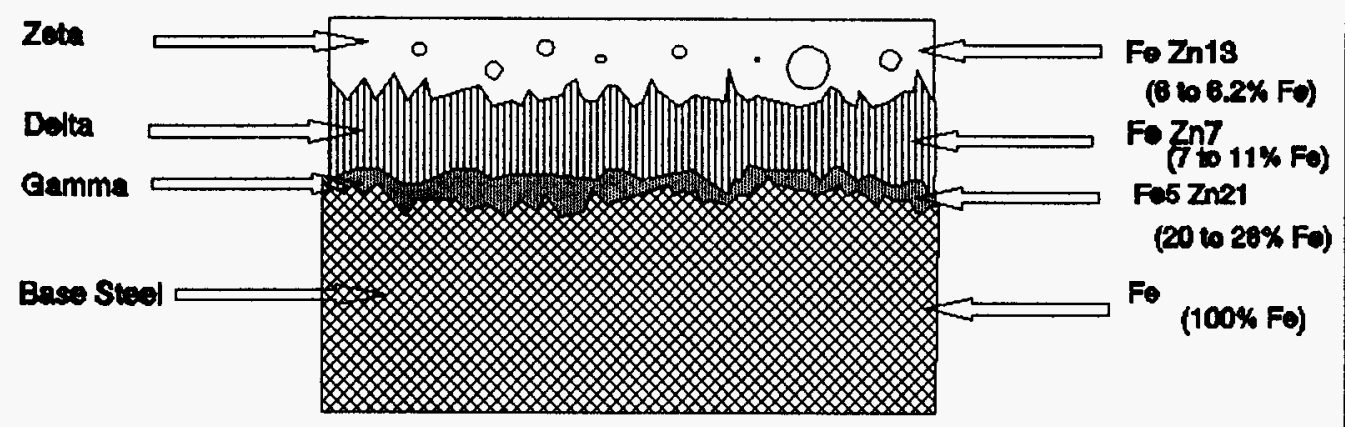

\section{Ideal Structure:}

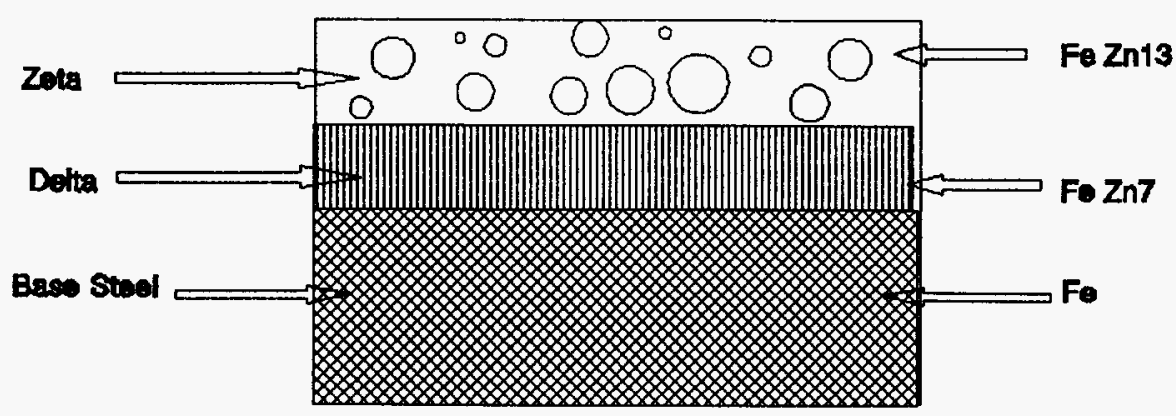


- introducing the preceding step's mathematical representation as a software augmentation to a DMC gauge installed at an operational galvanneal line,

- comparing the on-line gauge-generated phase distributions with off-line laboratory-measured phase distributions of galvanneal samples timetagged on the same production line,

- iterating until satisfactory agreement is reached.

The remainder of this section demonstrates that following this technical approach validated the performance of the augmented gauge.

\subsection{Creating Galvanneal Sample and Training Sets ${ }^{1}$}

Project E's initial task was to acquire a sufficiently realistic and diverse ensemble of galvanneal samples. After consultation among the project participants, Inland Steel chose to produce the samples at its pilot coating facility, an approximately one third scale model of an actual galvannealing line. As shown in Figure 3, this facility contains an electrolytic cleaning section, an annealing furnace, and a zinc pot with coating control knives. An induction furnace mounted over the zinc pot heat-treats the coated strip and promotes the zinc/iron reaction. Inland's strategy for achieving the sample ensemble was systematic variation of three parameters known to influence galvanneal phase structure:

- substrate chemistry,

- zinc coating weight,

- degree of zinc/iron alloying.

The three substrate types are extra-low-carbon (ELC), interstitial-free (IF), and rephosphorized (Rephos) corresponding to chemistries with low carbon, niobium-plus- titanium ultra low carbon, and niobium-plus-titanium-plusphosphorus ultra low carbon, respectively. The six values of zinc coating weight range from 30 to $110 \mathrm{~g} / \mathrm{m}^{2}$, and the six values of zinc/iron alloying range from

\footnotetext{
${ }^{1}$ The content of this subsection is drawn heavily from Reference 2.
} 


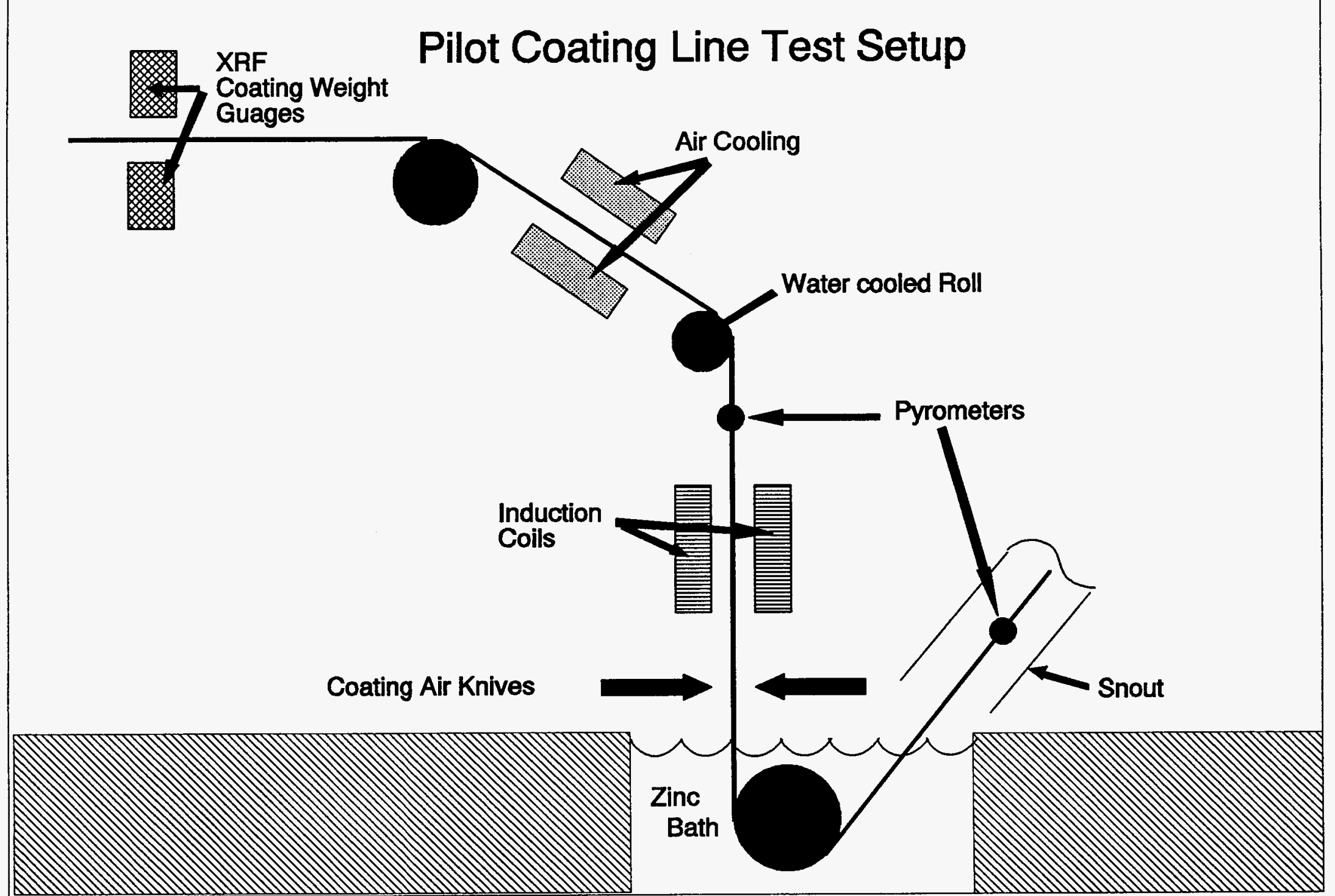


unalloyed to severely over-alloyed. This systematic variation of the three parameters clearly corresponds to 108 different combinations of galvanneal properties; multiple duplications of each combination served to guarantee a sufficient quantity of samples.

The empirical variation of parameters was achieved as follows. The choice of substrate type was clearly direct. The desired zinc coating weight was achieved by adjusting the air knife pressure and distance while keeping constant the speed of the strip as it ran through the line. The desired degree of zinc/iron alloying was achieved by varying the induction furnace power; the visual appearance of the strip surface leaving the induction furnace was the indicator of alloying. During the galvanneal runs, Inland recorded several parameters critical to the zinc/iron reaction. These recorded parameters included

- line speed,

- zinc pot temperature,

- zinc pot chemistry (percentages of aluminum and iron),

- temperature of the strip as it enters the pot,

- temperature of the strip as it leaves galvanneal furnace.

For each of the processing conditions, the test coils were cut up into ten meter segments. A set of sample coupons was punched from each coil segment, and four duplicate coupons were selected from each sample set. Three of the four coupons were retained at Inland, while the fourth was sent to DMC for nondestructive tests and thence to JPL for destructive tests, including the measurement of the galvanneal phase distribution of each sample.

At AISI's direction, the report summarizing JPL's work on Project E is included in this report. It appears as Appendix I below. JPL measured the galvanneal samples' average phase thicknesses from optical photomicrographs of etched sample cross-sections taken at thousand-fold magnification. The JPLmeasured phase distributions appear as Table 6 in Appendix I.

Before sending the Inland-produced samples to JPL, DMC examined the samples in the laboratory version of the DMC800 Zinc/Iron Coating Gauge which 
is discussed in the next subsection. Suffice it here to note that both versions of this gauge measure the galvanneal coating weight and iron content. In the mathematical parameter-space defined by these two measurable quantities, Figure 4 and 5 show the region filled by the Inland-produced samples' idealized and realized ensembles, respectively. The two regions clearly exhibit strong overlap.

\subsection{Hardware Base: DMC800 Zinc/Iron Coating Gauge ${ }^{2}$}

The hardware starting point for this project is the commercially available DMC800 Zinc/Iron Coating Gauge. This gauge provides

- simultaneous measurement of coating weight and Iron Migration Index (IMI)/percent iron,

- rugged scanning frames for measuring both sides of the product,

- update rates of 25 milliseconds for all four measurements (i.e., coating weight and percent iron, both sides),

- coating weight and percent iron profiles across the width of the product,

- "pure zinc" mode for galvanized coatings

- proven accuracy, repeatability, reliability, and safety.

The gauge uses the fundamental principles of $x$-ray fluorescence, together with high-intensity $x$-ray sources, as the basis for the measurements. The resulting signals are strong and suitable for achieving fast response times, high signal-to-noise ratios, and excellent accuracy. By combining a unique measurement geometry with intense photon fluxes and optimized energy levels, DMC's patented approach enhances the fluorescence of iron in the galvanneal coating and suppresses the iron fluorescence signal from the steel substrate. The gauge has two measuring heads, one for each side of the strip. Each measurement head contains two x-ray sources and two $x$-ray fluorescence sensors. One source/detector pair is designated as the "Zinc head" and the other is designated the "Iron head." Figures 6 and 7 illustrate the physics underlying the Zinc and Iron heads, respectively. The Zinc head uses normal (i.e.,

\footnotetext{
${ }^{2}$ The content of this subsection is drawn heavily from Reference 3.
} 


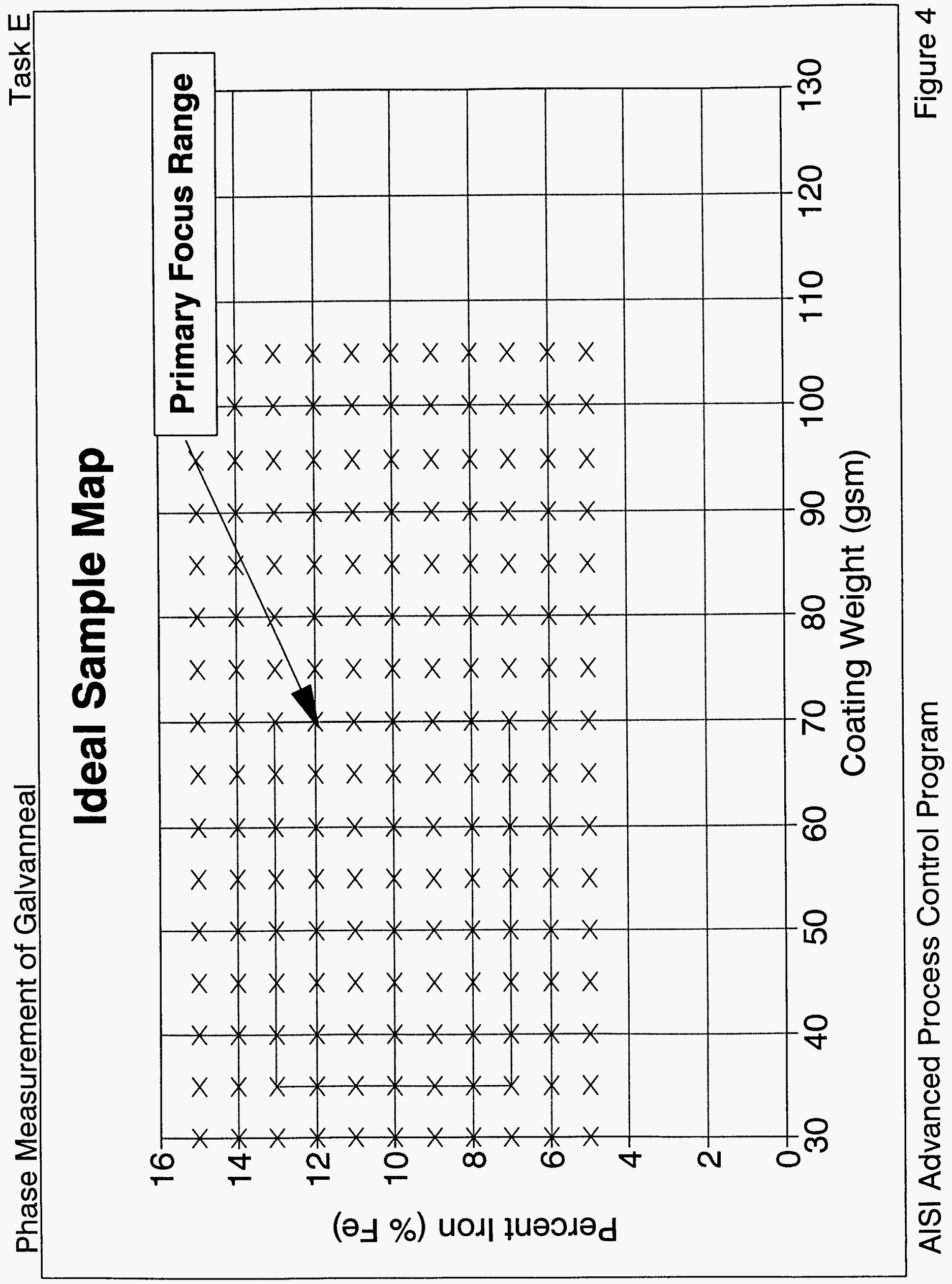




\section{Project Sample Map}

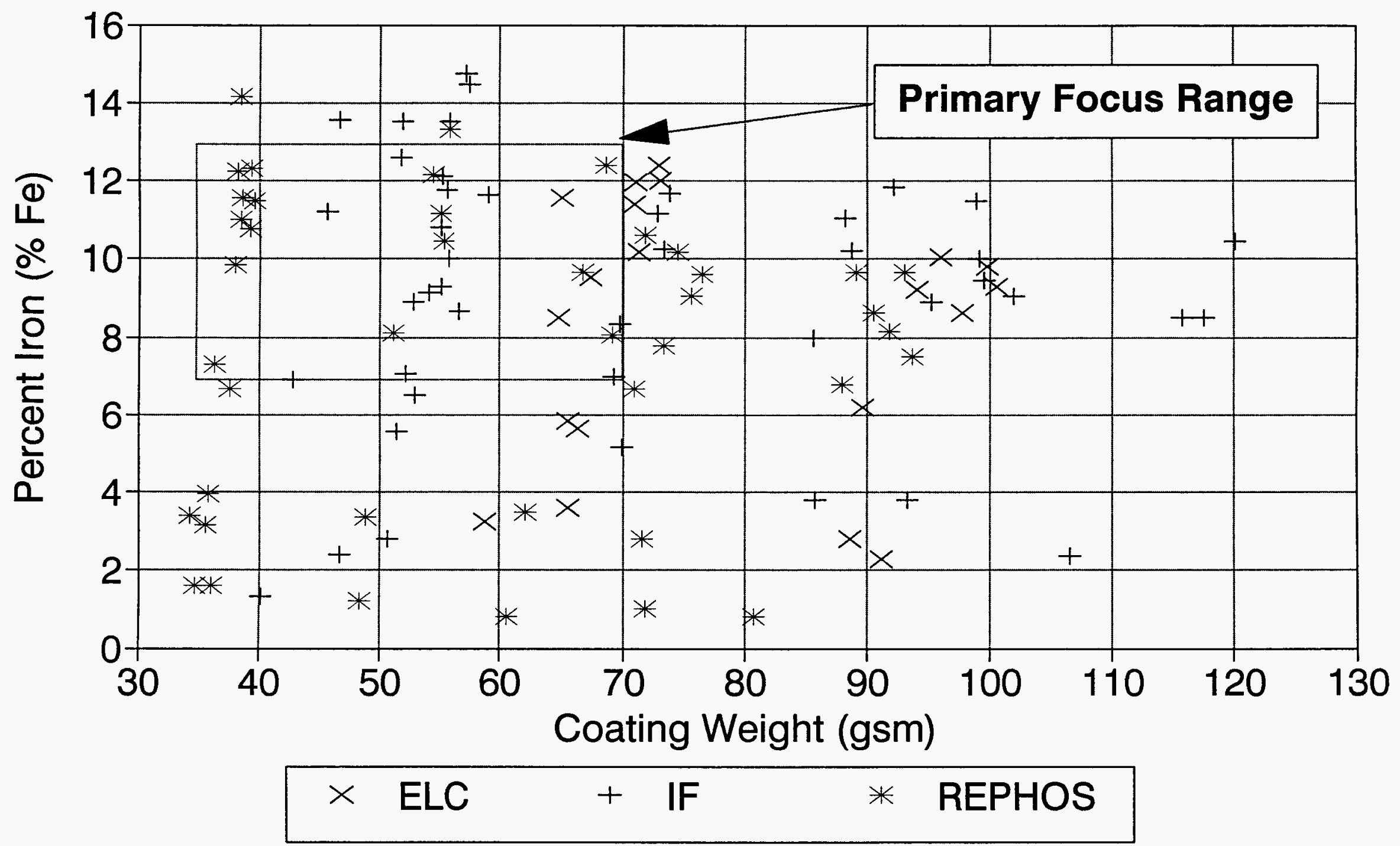




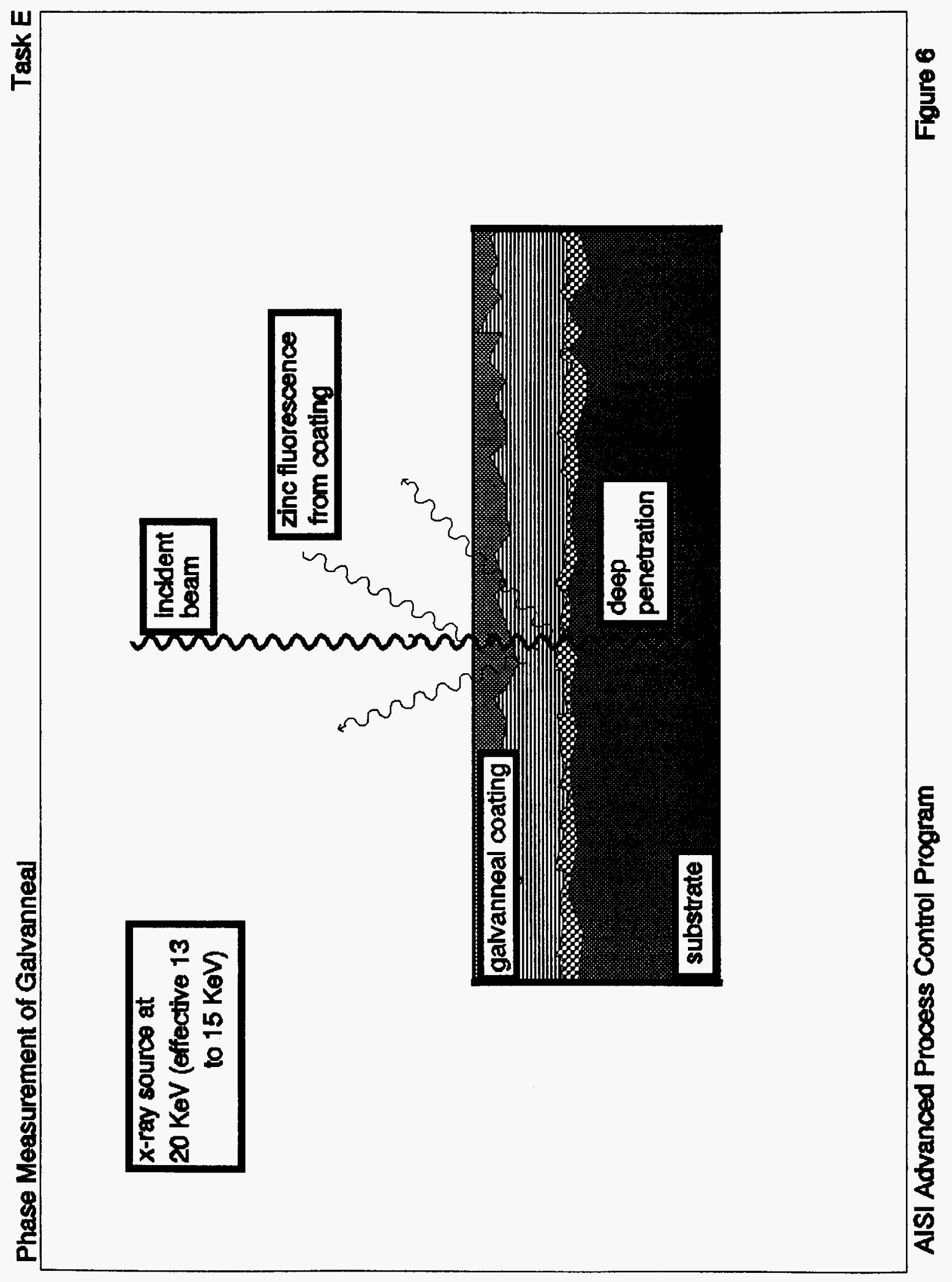




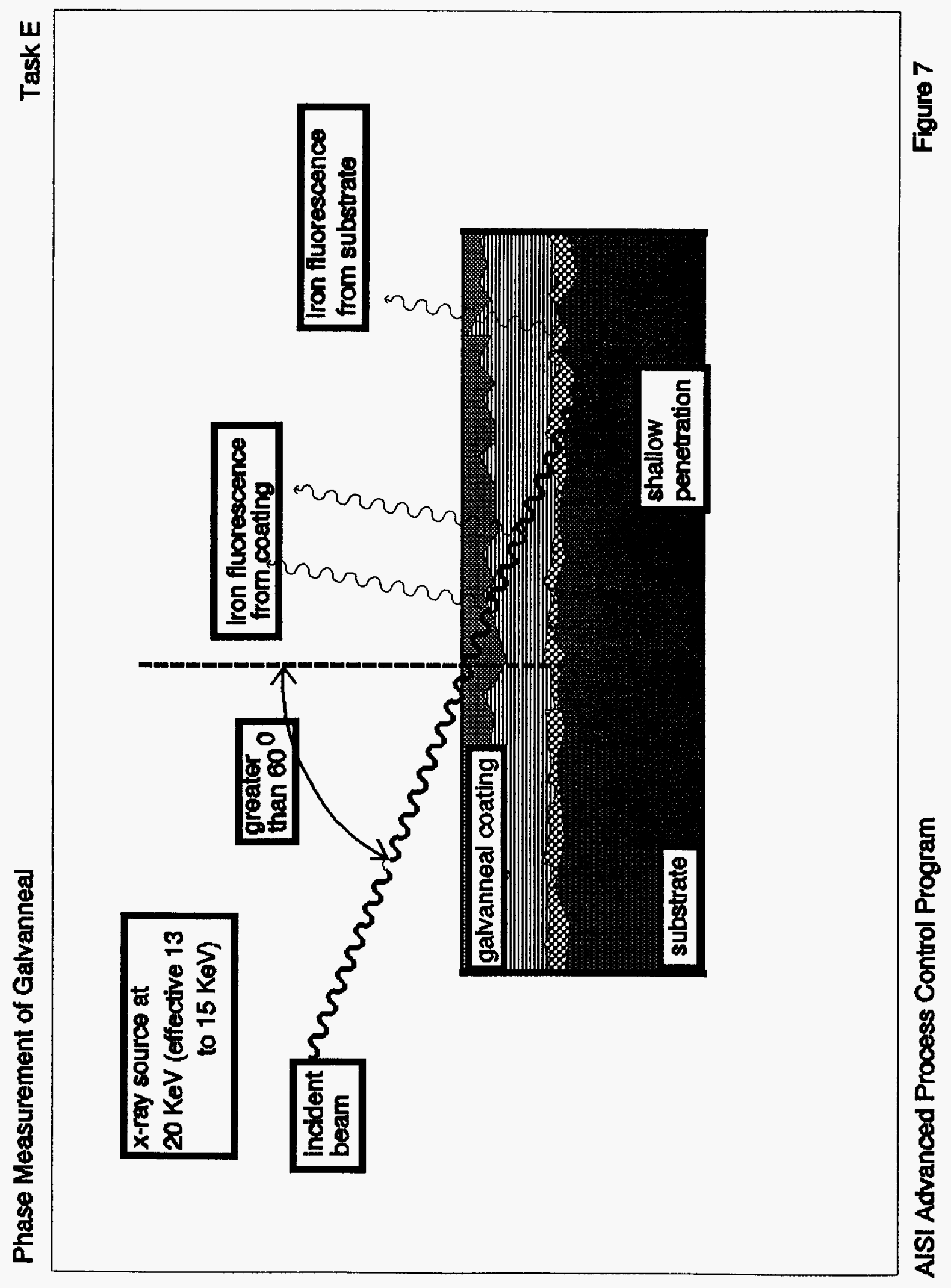


perpendicular to the coating) incidence and a differential annular ion chamber to detect fluorescence preferentially from zinc. The Iron head uses incidence widely separated from normal and a differential annular ion chamber to detect fluorescence preferentially from iron. When the outputs of the two source/detector pairs are jointly processed in the gauge's computer subsystem, the coating weight and percent iron can be inferred accurately in real-time, with sufficient speed and resolution to generate profiles down the length of the strip and across the width of the strip. Figure 8 illustrates a typical gauge installation. Appendix II presents a more detailed description and explanation of the DMC800 Zinc/Iron Coating Gauge's operation.

A sample's dimensional voltage output from the Zinc or Iron head can be rendered dimensionless (and lying between zero and one) via normalizing with respect to the output from bare zinc or bare iron (i.e., low carbon steel). Thus, for each type of detector, the relative voltage is given as

$$
\left(\mathrm{V}_{\text {sample }}-\mathrm{V}_{\min }\right) /\left(\mathrm{V}_{\max }-\mathrm{V}_{\min }\right)
$$

where $\mathrm{V}_{\text {sample }}$ is the detector output from the sample and $\mathrm{V}_{\max }$ and $\mathrm{V}_{\min }$ are the detector outputs from the same or opposed atomic-element, respectively, as the detector. Thus, for example, for the Zinc head, the maximum and minimum voltages arise from bare zinc and bare iron, respectively; for the Iron head, the results are simply transposed. The relative zinc and iron voltages were recorded for each Inland-produced sample when the sample was observed in the laboratory version of the DMC800 Zinc/Iron Coating Gauge.

\subsection{Software Augmentation}

The preceding subsections have described how measurements of the phase distribution and of the relative zinc and iron voltage were made on each Inlandproduced sample and hence provide a training-set basis for a predictive mathematical representation. The theory of neural networks provides the framework used by DMC for developing such a representation. Within this project, any specific numerical embodiment of the underlying mathematical representation was called a "math model." All such math models share the same generic conceptual framework and differ only in the numerical values assigned to their constituent parameters. As with any pattern recognition scheme, a "trainingset" is needed in order to flesh out the numerics of the representation's decision 


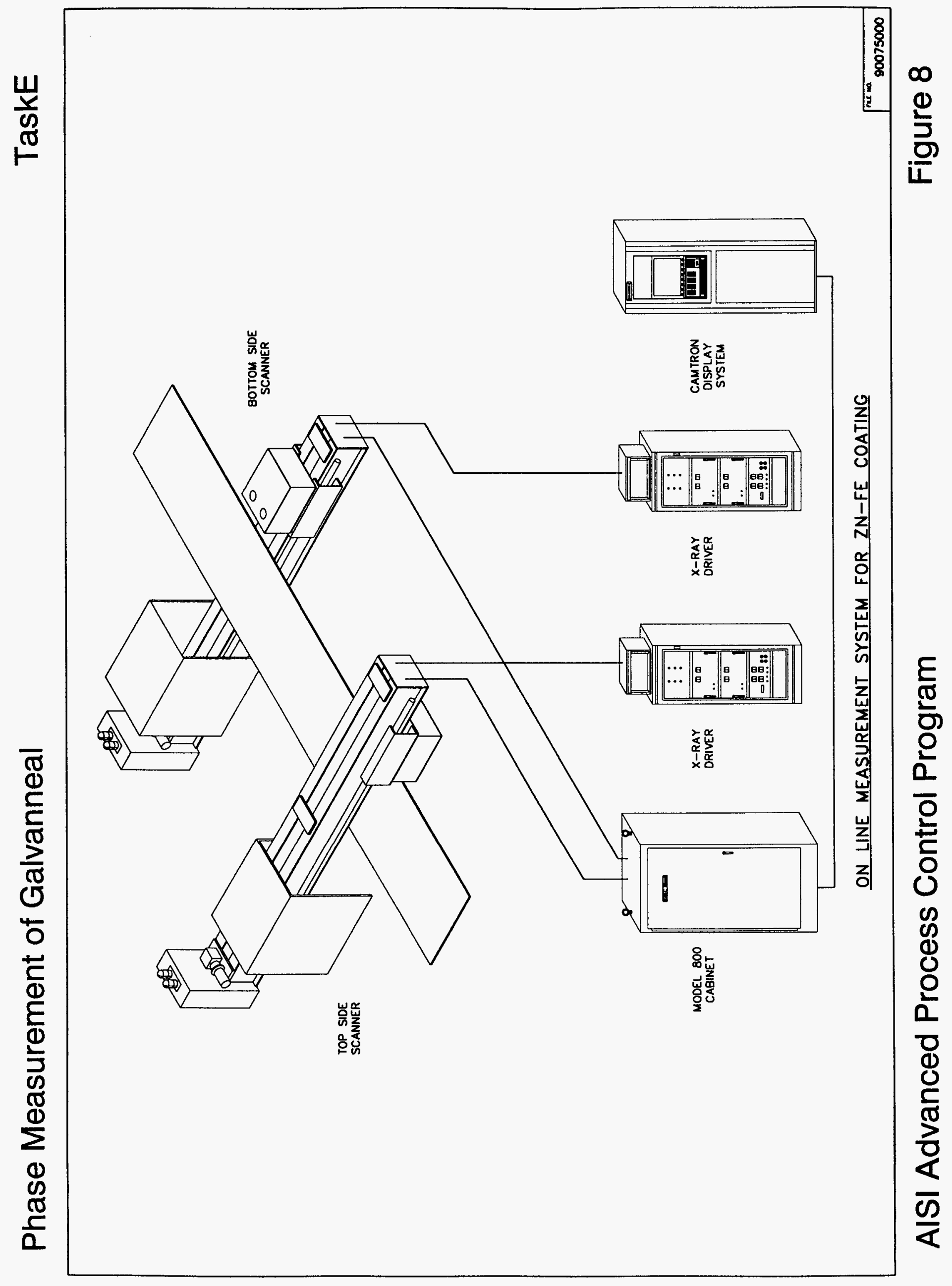


logic. All the training-sets considered during this phase of the project originated as subsets of the total population of Inland-produced samples. Program reviews produced a consensus that any candidate training-set should discard every sample that contained a coating phase consisting of pure zinc. Two training-sets meeting this criterion were created, containing seventy-four and sixty-six samples, respectively; the smaller set reflects discarding eight additional questionable samples. For each training-set, the mathematical representation's input and output parameters for each sample consisted of the two DMC-measured relative voltages described above and the three JPL-measured relative phases, respectively. The sole additional input property assigns each sample to one of the three mutually exclusive classes (viz., extra-low-carbon, interstitial-free, and rephosphorized) of galvanneal substrate; this property is both fundamental and static, inasmuch as it doesn't change during a production run. The resulting numerical incarnations of DMC's mathematical representation were called Math Models 1 and 2 according as the larger or smaller, respectively, of the two training-sets had served to characterize the model's numerics completely.

Although the theory of neural networks originated in a biological and neurophysiological context, it has long since been incorporated into mainstream mathematics. ${ }^{3}$ This subsection presents relevant rudiments of the theory in sufficient generality for this project: all variables are dimensionless with absolute value not exceeding one; input and output variables are positive; only one hidden layer is present. Within these restrictions, the following formalism applies.

There are three classes of nodes:

- $M$ Input Variables: $r_{1}, r_{2}, \ldots, r_{M}$

- $N$ Hidden Variables: $s_{1}, s_{2}, \ldots ., s_{N}$

- P Output Variables: $t_{1}, t_{2}, \ldots, t_{P}$

where $M, N$, and $P$ are positive integers.

\footnotetext{
${ }^{3}$ Topical confirmation comes from the Massachusetts Institute of Technology 1996 Summer Session Bulletin, announcing two programs numbered 2.15s and 6.95s and entitled Neural Networks for Nonlinear Estimation and Control and Intelligent Decision and Control with Neural Networks, respectively.
} 
In all the math models considered here, the input variables include the relative zinc and iron voltages and the three binary variables corresponding to the three substrate types; consequently, $M$ is 5 or more. The number of hidden variables in the single hidden layer is always 5 . The number of output variable is always 3 , corresponding to the three galvanneal phases.

The function $\mathrm{H}$ is defined via

$$
H(z) \equiv[1+\exp (-z)]^{-1} \text { where }-\infty<z<\infty
$$

Clearly, the function $\mathrm{H}$ is intrinsically positive and has the generic "sigmoid" property of increasing monotonically from 0 through $1 / 2$ to 1 , as its argument increases from $-\infty$ through 0 to $\infty$.

The neural network formalism is couched in terms of the real-valued coefficients defined - for $1 \leq \mathrm{i} \leq \mathrm{M}, 1 \leq \mathrm{j} \leq \mathrm{N}, 1 \leq \mathrm{k} \leq \mathrm{P}$ - via

$$
\begin{aligned}
& \mu_{i j} \equiv \text { coupling-coefficient between input variable } r_{i} \text { and hidden variable } s_{j} \\
& v_{j k} \equiv \text { coupling-coefficient between hidden variable } s_{j} \text { and output variable } t_{k} \\
& \sigma_{j} \equiv \text { bias-coefficient of hidden variable } s_{j} \\
& \tau_{k} \equiv \text { bias-coefficient of output variable } t_{k}
\end{aligned}
$$

The total number, $\mathrm{Q}$, of such parameters is clearly given as

$$
\mathrm{Q}=\mathrm{MN}+\mathrm{N} \mathrm{P}+\mathrm{N}+\mathrm{P}=(\mathrm{M}+1) \mathrm{N}+(\mathrm{N}+1) \mathrm{P}=\mathrm{P}+(\mathrm{M}+\mathrm{P}+1) \mathrm{N}
$$

The integers $\mathrm{M}, \mathrm{N}$, and $\mathrm{P}$ are 5, 5, and 3, respectively, for DMC's Math Models 1 and 2, and are 6, 5, and 3, respectively, for DMC's Composite Math Model. Hence $Q$ is 48 for DMC's Models 1 and 2, and is 53 for DMC's Composite Model.

The variables are interrelated - for $1 \leq \mathrm{j} \leq \mathrm{N}, 1 \leq \mathrm{k} \leq \mathrm{P}-$ via 


$$
\begin{aligned}
& \mathrm{s}_{\mathrm{j}}=\mathrm{H}\left(\sigma_{\mathrm{j}}+\sum_{i=1}^{M} \mu_{i j} r_{i}\right) \\
& \mathrm{t}_{\mathrm{k}}=\mathrm{H}\left(\tau_{\mathrm{k}}+\sum_{j=1}^{N} v_{j k} s_{j}\right)
\end{aligned}
$$

and thence by

$$
\mathrm{t}_{\mathrm{k}}=\mathrm{H}\left(\tau_{\mathrm{k}}+\sum_{j=1}^{N} v_{j k} \mathrm{H}\left(\sigma_{\mathrm{j}}+\sum_{i=1}^{M} \mu_{i j} r_{i}\right)\right)
$$

Since the galvanneal's relative phases must sum to one, this project's output variables are renormalized and given - for $1 \leq \mathrm{k} \leq \mathrm{P}$ - as the phases $\Phi_{\mathrm{k}}$ via

$$
\Phi_{\mathrm{k}}=\frac{\mathrm{H}\left(\tau_{\mathrm{k}}+\sum_{j=1}^{N} v_{j k} \mathrm{H}\left(\sigma_{\mathrm{j}}+\sum_{i=1}^{M} \mu_{i j} r_{i}\right)\right)}{\sum_{k=1}^{P} \mathrm{H}\left(\tau_{\mathrm{k}}+\sum_{j=1}^{N} v_{j k} \mathrm{H}\left(\sigma_{\mathrm{j}}+\sum_{i=1}^{M} \mu_{i j} r_{i}\right)\right)}
$$

In order to flesh out the numerics of the neural network representation, it is necessary to establish values for the total number $Q$ of coefficients described above. The fundamental approach (Refs. 4,5 ) is to determine the coefficients so as to minimize the separation, summed over all samples in the training set, between the measured phases (i.e., the left-hand side of the preceding equation) and the phases calculated (via the right-hand side of the preceding equation) as a function of the observed input variables and of the to-be-determined coefficients. In the parlance of neural networks, the solution for the coefficients is achieved by back propagation, frequently via some variant of the so-called Widrow-Hoff algorithm.(Refs. 4, 5)

By proceeding in this fashion, the numerics were established for each of DMC's math models. Table 1 summarizes Math Model 1's training-set, consisting of seventy-four samples. In this and subsequent tables, 


\begin{tabular}{|c|c|c|c|c|c|c|c|c|}
\hline & $\mathbf{A}$ & 8 & c & D & $\mathbf{E}$ & $\mathbf{F}$ & G & H \\
\hline \multicolumn{9}{|l|}{1} \\
\hline \multicolumn{9}{|c|}{1} \\
\hline 3 & & \multicolumn{6}{|c|}{ Table 1. Tralning-Sel for Model 1} & \\
\hline 4 & & & & & & & & \\
\hline 5 & & & & & & & & \\
\hline 6 & & & & & & Measured & Measured & Measured \\
\hline 7 & $\mathrm{~V} / \mathrm{Zn}$ & V/Fe & EC & $F$ & FE+1 & Zota & Delta & Gamma \\
\hline 8 & & & & & & & & \\
\hline 9 & 0.079 & 0.859 & 0 & 1 & 0 & 0.230 & 0.750 & 0.016 \\
\hline 10 & 0.161 & 0.733 & 0 & 1 & 0 & 0.380 & 0.571 & 0.048 \\
\hline 11 & 0.161 & 0.765 & 0 & 0 & 1 & 0.300 & 0.679 & 0.019 \\
\hline 12 & 0.164 & 0.755 & 0 & 1 & 0 & 0.270 & 0.692 & 0.039 \\
\hline 13 & 0.168 & 0.759 & 0 & 0 & 1 & 0.410 & 0.571 & 0.020 \\
\hline 14 & 0.170 & 0.715 & 1 & 0 & 0 & 0.330 & 0.583 & 0.083 \\
\hline 15 & 0.171 & 0.765 & 0 & 0 & 1 & 0.230 & 0.755 & 0.019 \\
\hline 16 & 0.178 & 0.757 & 0 & 0 & 1 & 0.390 & 0.590 & 0.016 \\
\hline 17 & 0.180 & 0.720 & 0 & 0 & 1 & 0.490 & 0.490 & 0.020 \\
\hline 18 & 0.182 & 0.749 & 0 & 0 & 1 & 0.330 & 0.653 & 0.020 \\
\hline 19 & 0.188 & 0.761 & 0 & 1 & 0 & 0.040 & 0.943 & 0.019 \\
\hline 20 & 0.189 & 0.755 & 1 & 0 & 0 & 0.000 & 0.960 & 0.040 \\
\hline 21 & 0.191 & 0.767 & 0 & 1 & 0 & 0.200 & 0.767 & 0.033 \\
\hline 22 & 0.191 & 0.762 & 1 & 0 & 0 & 0.000 & 0.963 & 0.037 \\
\hline 23 & 0.192 & 0.722 & 0 & 0 & 1 & 0.440 & 0.533 & 0.022 \\
\hline 24 & 0.193 & 0.761 & 1 & 0 & 0 & 0.000 & 0.923 & 0.077 \\
\hline 25 & 0.193 & 0.768 & 0 & 1 & 0 & 0.000 & 0.941 & 0.059 \\
\hline 26 & 0.194 & 0.765 & 0 & 1 & 0 & 0.150 & 0.830 & 0.019 \\
\hline 27 & 0.195 & 0.748 & 0 & 0 & 1 & 0.280 & 0.702 & 0.018 \\
\hline 28 & 0.198 & 0.711 & 0 & 1 & 0 & 0.330 & 0.611 & 0.056 \\
\hline 29 & 0.206 & 0.714 & 0 & 0 & 1 & 0.330 & 0.653 & 0.020 \\
\hline 30 & 0.212 & 0.743 & 0 & 1 & 0 & 0.040 & 0.885 & 0.077 \\
\hline 31 & 0.213 & 0.710 & 0 & 0 & 1 & 0.240 & 0.735 & 0.020 \\
\hline 32 & 0.213 & 0.743 & 1 & 0 & 0 & 0.100 & 0.867 & 0.033 \\
\hline 33 & 0.216 & 0.680 & 1 & 0 & 0 & 0.280 & 0.667 & 0.056 \\
\hline 34 & 0.219 & 0.698 & 0 & 0 & 1 & 0.270 & 0.711 & 0.022 \\
\hline 35 & 0.220 & 0.660 & 0 & 1 & 0 & 0.390 & 0.581 & 0.032 \\
\hline 36 & 0.221 & 0.658 & 0 & 0 & 1 & 0.300 & 0.700 & 0.000 \\
\hline 37 & 0.221 & 0.680 & 0 & 0 & 1 & 0.270 & 0.682 & 0.046 \\
\hline 38 & 0.224 & 0.689 & 1 & 0 & 0 & 0.150 & 0.750 & 0.100 \\
\hline 39 & 0.227 & 0.674 & 0 & 0 & 1 & 0.270 & 0.682 & 0.046 \\
\hline 40 & 0.227 & 0.725 & 0 & 1 & 0 & 0.040 & 0.875 & 0.083 \\
\hline 41 & 0.227 & 0.702 & 0 & 1 & 0 & 0.140 & 0.818 & 0.046 \\
\hline 42 & 0.230 & 0.730 & 0 & 1 & 0 & 0.000 & 0.893 & 0.107 \\
\hline 43 & 0.234 & 0.691 & 0 & 1 & 0 & 0.000 & 0.952 & 0.048 \\
\hline 44 & 0.235 & 0.707 & 0 & 1 & 0 & 0.040 & 0.875 & 0.083 \\
\hline 45 & 0.236 & 0.683 & 1 & 0 & 0 & 0.020 & 0.909 & 0.068 \\
\hline 46 & 0.244 & 0.687 & 0 & 1 & 0 & 0.000 & 0.900 & 0.100 \\
\hline 47 & 0.251 & 0.641 & 0 & 1 & 0 & 0.250 & 0.688 & 0.063 \\
\hline 48 & 0.252 & 0.673 & 1 & 0 & 0 & 0.020 & 0.878 & 0.098 \\
\hline 40 & 0.252 & 0.667 & 0 & 0 & 1 & 0.200 & 0.780 & 0.024 \\
\hline 50 & 0.253 & 0.623 & 0 & 1 & 0 & 0.250 & 0.688 & 0.063 \\
\hline 51 & 0.261 & 0.671 & 0 & 1 & 0 & 0.000 & 0.900 & 0.100 \\
\hline 52 & 0.262 & 0.671 & 1 & 0 & 0 & 0.000 & 0.900 & 0.100 \\
\hline 53 & 0.263 & 0.659 & 1 & 0 & 0 & 0.000 & 0.900 & 0.100 \\
\hline 54 & 0.263 & 0.623 & 0 & 1 & 0 & 0.380 & 0.563 & 0.063 \\
\hline 55 & 0.264 & 0.671 & 1 & 0 & 0 & 0.000 & 0.909 & 0.091 \\
\hline 56 & 0.267 & 0.630 & 0 & 1 & 0 & 0.190 & 0.750 & 0.063 \\
\hline 57 & 0.268 & 0.668 & 1 & 0 & 0 & 0.000 & 0.900 & 0.100 \\
\hline 58 & 0.269 & 0.613 & 0 & 1 & 0 & 0.130 & 0.813 & 0.063 \\
\hline 59 & 0.274 & 0.626 & 0 & 1 & 0 & 0.130 & 0.800 & 0.067 \\
\hline 60 & 0.279 & 0.632 & 0 & 1 & 0 & 0.060 & 0.848 & 0.091 \\
\hline 61 & 0.280 & 0.597 & 0 & 0 & 1 & 0.250 & 0.714 & 0.036 \\
\hline 62 & 0.286 & 0.607 & 0 & 1 & 0 & 0.060 & 0.875 & 0.063 \\
\hline 63 & 0.293 & 0.595 & 0 & 0 & 1 & 0.190 & 0.750 & 0.063 \\
\hline 84 & 0.298 & 0.599 & 0 & 0 & 1 & 0.120 & 0.808 & 0.077 \\
\hline 65 & 0.300 & 0.599 & 0 & 1 & 0 & 0.000 & 0.875 & 0.125 \\
\hline 66 & 0.306 & 0.598 & 0 & 1 & 0 & 0.000 & 0.844 & 0.156 \\
\hline 67 & 0.308 & 0.592 & 0 & 1 & 0 & 0.000 & 0.844 & 0.156 \\
\hline 68 & 0.309 & 0.590 & 0 & 0 & 1 & 0.110 & 0.750 & 0.143 \\
\hline 69 & 0.316 & 0.589 & 0 & 0 & 1 & 0.110 & 0.750 & 0.143 \\
\hline 70 & 0.316 & 0.573 & 0 & 1 & 0 & 0.000 & 0.917 & 0.083 \\
\hline 71 & 0.318 & 0.556 & 0 & 1 & 0 & 0.000 & 0.917 & 0.083 \\
\hline 72 & 0.332 & 0.562 & 0 & 1 & 0 & 0.000 & 0.821 & 0.179 \\
\hline 73 & 0.336 & 0.551 & 0 & 1 & 0 & 0.000 & 0.821 & 0.179 \\
\hline 74 & 0.348 & 0.489 & 0 & 0 & 1 & 0.500 & 0.500 & 0.000 \\
\hline 75 & 0.358 & 0.499 & 0 & 0 & 1 & 0.160 & 0.600 & 0.040 \\
\hline 76 & 0.359 & 0.505 & 0 & 0 & 1 & 0.130 & 0.833 & 0.042 \\
\hline 77 & 0.362 & 0.494 & 0 & 0 & 1 & 0.380 & 0.571 & 0.048 \\
\hline 78 & 0.368 & 0.498 & 0 & 0 & 1 & 0.130 & 0.833 & 0.042 \\
\hline 79 & 0.371 & 0.492 & 0 & 0 & 1 & 0.190 & 0.750 & 0.063 \\
\hline 80 & 0.371 & 0.497 & 0 & 0 & 1 & 0.150 & 0.800 & 0.050 \\
\hline 81 & 0.381 & 0.486 & 0 & 0 & 1 & 0.150 & 0.800 & 0.050 \\
\hline 82 & 0.385 & 0.491 & 0 & 0 & 1 & 0.140 & 0.727 & 0.136 \\
\hline
\end{tabular}


Table 2. Neural Network Coupling- and Bias-Coefficient Arrays for Model 1

\section{From Input Layer to Hidden Layer:}

\begin{tabular}{|c|c|c|c|c|c|}
\cline { 2 - 6 } \multicolumn{1}{c|}{} & \multicolumn{5}{c|}{ Hidden Layer } \\
\hline Input Layer & 1 & 2 & 3 & 4 & 5 \\
\hline 1 & 16.093866 & 1.672538 & 24.948534 & 31.813299 & -16.605635 \\
\hline 2 & 3.048830 & 20.253468 & 43.688843 & 1.154431 & -25.813431 \\
\hline 3 & -2.999765 & -2.203046 & -6.015329 & -8.756530 & 3.077070 \\
\hline 4 & -3.951983 & 2.159524 & -7.516699 & -8.586378 & 3.043841 \\
\hline 5 & -5.414382 & -3.800123 & -7.864215 & -2.653580 & 3.248891 \\
\hline
\end{tabular}

\begin{tabular}{|c|c|c|c|c|c|}
\cline { 2 - 6 } \multicolumn{1}{c|}{} & \multicolumn{5}{c|}{ Hidden Layer } \\
\cline { 2 - 6 } \multicolumn{1}{c|}{} & 1 & 2 & 3 & 4 & 5 \\
\hline Bias & -11.050628 & -8.663224 & -20.990395 & -13.799554 & 11.168233 \\
\hline
\end{tabular}

\section{From Hidden Layer to Output Layer:}

\begin{tabular}{|c|c|c|c|}
\cline { 2 - 4 } \multicolumn{1}{c|}{} & \multicolumn{3}{c|}{ Output Layer } \\
\hline $\begin{array}{c}\text { Hidden } \\
\text { Layer }\end{array}$ & 1 & 2 & 3 \\
\hline 1 & -2.661684 & 3.076196 & -6.635210 \\
\hline 2 & -3.873622 & 1.673202 & 1.560143 \\
\hline 3 & -6.151527 & 2.373915 & 3.086006 \\
\hline 4 & -1.946271 & 0.943076 & 0.782466 \\
\hline 5 & -0.052203 & -8.391353 & -2.116872 \\
\hline
\end{tabular}

\begin{tabular}{|c|c|c|c|}
\cline { 2 - 4 } \multicolumn{1}{c|}{} & \multicolumn{3}{c|}{ Output Layer } \\
\cline { 2 - 4 } \multicolumn{1}{c|}{} & 1 & 2 & 3 \\
\hline Bias & 1.818682 & 0.028800 & -4.144551 \\
\hline
\end{tabular}




\begin{tabular}{|c|c|c|c|c|c|c|c|c|}
\hline & A & B & $\mathbf{C}$ & D & $\mathbf{E}$ & $\mathbf{F}$ & $\mathbf{G}$ & $\mathrm{H}$ \\
\hline 1 & & & & & & & & \\
\hline 2 & & & & & & & & \\
\hline 3 & & \multicolumn{6}{|c|}{ Table 4. Training-Set for Model 2} & \\
\hline 4 & & & & & & & & \\
\hline 5 & & & & & & & & \\
\hline 6 & & & & & & Measured & Measured & Measured \\
\hline 7 & $V / Z n$ & $\mathrm{~V} / \mathrm{F}_{\theta}$ & Ec & $\mathbf{F}$ & FEH & Zota & Delta & Gamma \\
\hline 8 & & & & & & & & \\
\hline 9 & 0.079 & 0.859 & 0 & 1 & 0 & 0.234 & 0.750 & 0.016 \\
\hline 10 & 0.161 & 0.765 & 0 & 0 & 1 & 0.302 & 0.679 & 0.019 \\
\hline 11 & 0.164 & 0.755 & 0 & 1 & 0 & 0.269 & 0.692 & 0.038 \\
\hline 12 & 0.168 & 0.759 & 0 & 0 & 1 & 0.408 & 0.571 & 0.020 \\
\hline 13 & 0.171 & 0.765 & 0 & 0 & 1 & 0.226 & 0.755 & 0.019 \\
\hline 14 & 0.176 & 0.757 & 0 & 0 & 1 & 0.393 & 0.590 & 0.016 \\
\hline 15 & 0.180 & 0.720 & 0 & 0 & 1 & 0.490 & 0.490 & 0.020 \\
\hline 16 & 0.182 & 0.749 & 0 & 0 & 1 & 0.327 & 0.653 & 0.020 \\
\hline 17 & 0.188 & 0.761 & 0 & 1 & 0 & 0.038 & 0.943 & 0.019 \\
\hline 18 & 0.189 & 0.755 & 1 & 0 & 0 & 0.000 & 0.960 & 0.040 \\
\hline 19 & 0.191 & 0.767 & 0 & 1 & 0 & 0.200 & 0.767 & 0.033 \\
\hline 20 & 0.191 & 0.762 & 1 & 0 & 0 & 0.000 & 0.963 & 0.037 \\
\hline 21 & 0.192 & 0.722 & 0 & 0 & 1 & 0.444 & 0.533 & 0.022 \\
\hline 22 & 0.193 & 0.761 & 1 & 0 & 0 & 0.000 & 0.923 & 0.077 \\
\hline 23 & 0.193 & 0.768 & 0 & 1 & 0 & 0.000 & 0.941 & 0.059 \\
\hline 24 & 0.194 & 0.765 & 0 & 1 & 0 & 0.151 & 0.830 & 0.019 \\
\hline 25 & 0.195 & 0.748 & 0 & 0 & 1 & 0.281 & 0.702 & 0.018 \\
\hline 26 & 0.206 & 0.714 & 0 & 0 & 1 & 0.327 & 0.653 & 0.020 \\
\hline 27 & 0.212 & 0.743 & 0 & 1 & 0 & 0.038 & 0.885 & 0.077 \\
\hline 28 & 0.213 & 0.710 & 0 & 0 & 1 & 0.245 & 0.735 & 0.020 \\
\hline 29 & 0.213 & 0.743 & 1 & 0 & 0 & 0.100 & 0.867 & 0.033 \\
\hline 30 & 0.219 & 0.698 & 0 & 0 & 1 & 0.267 & 0.711 & 0.022 \\
\hline 31 & 0.221 & 0.658 & 0 & 0 & 1 & 0.300 & 0.700 & 0.000 \\
\hline 32 & 0.221 & 0.680 & 0 & 0 & 1 & 0.273 & 0.682 & 0.045 \\
\hline 33 & 0.227 & 0.674 & 0 & 0 & 1 & 0.273 & 0.682 & 0.045 \\
\hline 34 & 0.227 & 0.725 & 0 & 1 & 0 & 0.042 & 0.875 & 0.083 \\
\hline 35 & 0.227 & 0.702 & 0 & 1 & 0 & 0.136 & 0.818 & 0.045 \\
\hline 36 & 0.230 & 0.730 & 0 & 1 & 0 & 0.000 & 0.893 & 0.107 \\
\hline 37 & 0.234 & 0.691 & 0 & 1 & 0 & 0.000 & 0.952 & 0.048 \\
\hline 38 & 0.235 & 0.707 & 0 & 1 & 0 & 0.042 & 0.875 & 0.083 \\
\hline 39 & 0.236 & 0.683 & 1 & 0 & 0 & 0.023 & 0.909 & 0.068 \\
\hline 40 & 0.244 & 0.687 & 0 & 1 & 0 & 0.000 & 0.900 & 0.100 \\
\hline 41 & 0.251 & 0.641 & 0 & 1 & 0 & 0.250 & 0.688 & 0.063 \\
\hline 42 & 0.252 & 0.673 & 1 & 0 & 0 & 0.024 & 0.878 & 0.098 \\
\hline 43 & 0.253 & 0.623 & 0 & 1 & 0 & 0.250 & 0.688 & 0.063 \\
\hline 44 & 0.261 & 0.671 & 0 & 1 & 0 & 0.000 & 0.900 & 0.100 \\
\hline 45 & 0.262 & 0.671 & 1 & 0 & 0 & 0.000 & 0.900 & 0.100 \\
\hline 46 & 0.263 & 0.659 & 1 & 0 & 0 & 0.000 & 0.900 & 0.100 \\
\hline 47 & 0.264 & 0.671 & 1 & 0 & 0 & 0.000 & 0.909 & 0.091 \\
\hline 48 & 0.267 & 0.630 & 0 & 1 & 0 & 0.188 & 0.750 & 0.063 \\
\hline 49 & 0.268 & 0.668 & 1 & 0 & 0 & 0.000 & 0.900 & 0.100 \\
\hline 50 & 0.269 & 0.613 & 0 & 1 & 0 & 0.125 & 0.813 & 0.063 \\
\hline 51 & 0.274 & 0.626 & 0 & 1 & 0 & 0.133 & 0.800 & 0.067 \\
\hline 52 & 0.279 & 0.632 & 0 & 1 & 0 & 0.061 & 0.848 & 0.091 \\
\hline 53 & 0.280 & 0.597 & 0 & 0 & 1 & 0.250 & 0.714 & 0.036 \\
\hline 54 & 0.286 & 0.607 & 0 & 1 & 0 & 0.063 & 0.875 & 0.063 \\
\hline 55 & 0.293 & 0.595 & 0 & 0 & 1 & 0.000 & 0.938 & 0.063 \\
\hline 56 & 0.298 & 0.599 & 0 & 0 & 1 & 0.115 & 0.808 & 0.077 \\
\hline 57 & 0.300 & 0.599 & 0 & 1 & 0 & 0.000 & 0.875 & 0.125 \\
\hline 58 & 0.306 & 0.598 & 0 & 1 & 0 & 0.000 & 0.844 & 0.156 \\
\hline 59 & 0.308 & 0.592 & 0 & 1 & 0 & 0.000 & 0.844 & 0.156 \\
\hline 60 & 0.309 & 0.590 & 0 & 0 & 1 & 0.107 & 0.750 & 0.143 \\
\hline 61 & 0.316 & 0.589 & 0 & 0 & 1 & 0.107 & 0.750 & 0.143 \\
\hline 62 & 0.316 & 0.573 & 0 & 1 & 0 & 0.000 & 0.917 & 0.083 \\
\hline 63 & 0.318 & 0.556 & 0 & 1 & 0 & 0.000 & 0.917 & 0.083 \\
\hline 64 & 0.332 & 0.562 & 0 & 1 & 0 & 0.000 & 0.821 & 0.179 \\
\hline 65 & 0.336 & 0.551 & 0 & 1 & 0 & 0.000 & 0.821 & 0.179 \\
\hline 66 & 0.348 & 0.489 & 0 & 0 & 1 & 0.500 & 0.500 & 0.000 \\
\hline 67 & 0.358 & 0.499 & 0 & 0 & 1 & 0.160 & 0.800 & 0.040 \\
\hline 68 & 0.359 & 0.505 & 0 & 0 & 1 & 0.125 & 0.833 & 0.042 \\
\hline 69 & 0.362 & 0.494 & 0 & 0 & 1 & 0.381 & 0.571 & 0.048 \\
\hline 70 & 0.368 & 0.498 & 0 & 0 & 1 & 0.125 & 0.833 & 0.042 \\
\hline 71 & 0.371 & 0.492 & 0 & 0 & 1 & 0.000 & 0.938 & 0.063 \\
\hline 72 & 0.371 & 0.497 & 0 & 0 & 1 & 0.150 & 0.800 & 0.050 \\
\hline 73 & 0.381 & 0.486 & 0 & 0 & 1 & 0.150 & 0.800 & 0.050 \\
\hline 74 & 0.385 & 0.491 & 0 & 0 & 1 & 0.000 & 0.864 & 0.136 \\
\hline
\end{tabular}


Table 5. Neural Network Coupling- and Bias-Coefficient Arrays for Model 2

\section{From Input Layer to Hidden Layer:}

\begin{tabular}{|c|c|c|c|c|c|}
\cline { 2 - 6 } \multicolumn{1}{c|}{} & \multicolumn{5}{c|}{ Hidden Layer } \\
\hline Input Layer & 1 & 2 & 3 & 4 & 5 \\
\hline 1 & -52.601749 & 28.082651 & 26.277493 & 13.104314 & 50.552052 \\
\hline 2 & -1.978796 & -15.683971 & -7.775424 & 59.295624 & 53.25642 \\
\hline 3 & 4.065629 & 0.32091 & 1.254817 & 3.303699 & -0.151049 \\
\hline 4 & 1.167607 & 2.625571 & 6.576689 & -11.655663 & -4.082536 \\
\hline 5 & 3.190143 & -5.19048 & -11.705255 & -17.609793 & -16.965797 \\
\hline
\end{tabular}

\begin{tabular}{|c|c|c|c|c|c|}
\cline { 2 - 6 } \multicolumn{1}{c|}{} & \multicolumn{5}{c|}{ Hidden Layer } \\
\cline { 2 - 6 } \multicolumn{1}{c|}{} & 1 & 2 & 3 & 4 & 5 \\
\hline Bias & 9.325702 & 0.458199 & -0.734695 & -31.335619 & -26.52951 \\
\hline
\end{tabular}

\section{From Hidden Layer to Output Layer:}

\begin{tabular}{|c|c|c|c|}
\cline { 2 - 4 } \multicolumn{1}{c|}{} & \multicolumn{3}{c|}{ Output Layer } \\
\hline $\begin{array}{c}\text { Hidden } \\
\text { Layer }\end{array}$ & 1 & 2 & 3 \\
\hline 1 & 2.895404 & -1.829693 & -3.329598 \\
\hline 2 & -7.874425 & 4.109768 & 4.492254 \\
\hline 3 & 6.25185 & -3.558452 & -4.312635 \\
\hline 4 & -6.722804 & 3.991816 & 3.560675 \\
\hline 5 & -6.162772 & 3.904595 & 9.060319 \\
\hline
\end{tabular}

\begin{tabular}{|c|c|c|c|}
\cline { 2 - 4 } \multicolumn{1}{c|}{} & \multicolumn{3}{c|}{ Output Layer } \\
\cline { 2 - 4 } \multicolumn{1}{c|}{} & 1 & 2 & 3 \\
\hline Bias & 3.906097 & -2.269504 & -10.89089 \\
\hline
\end{tabular}




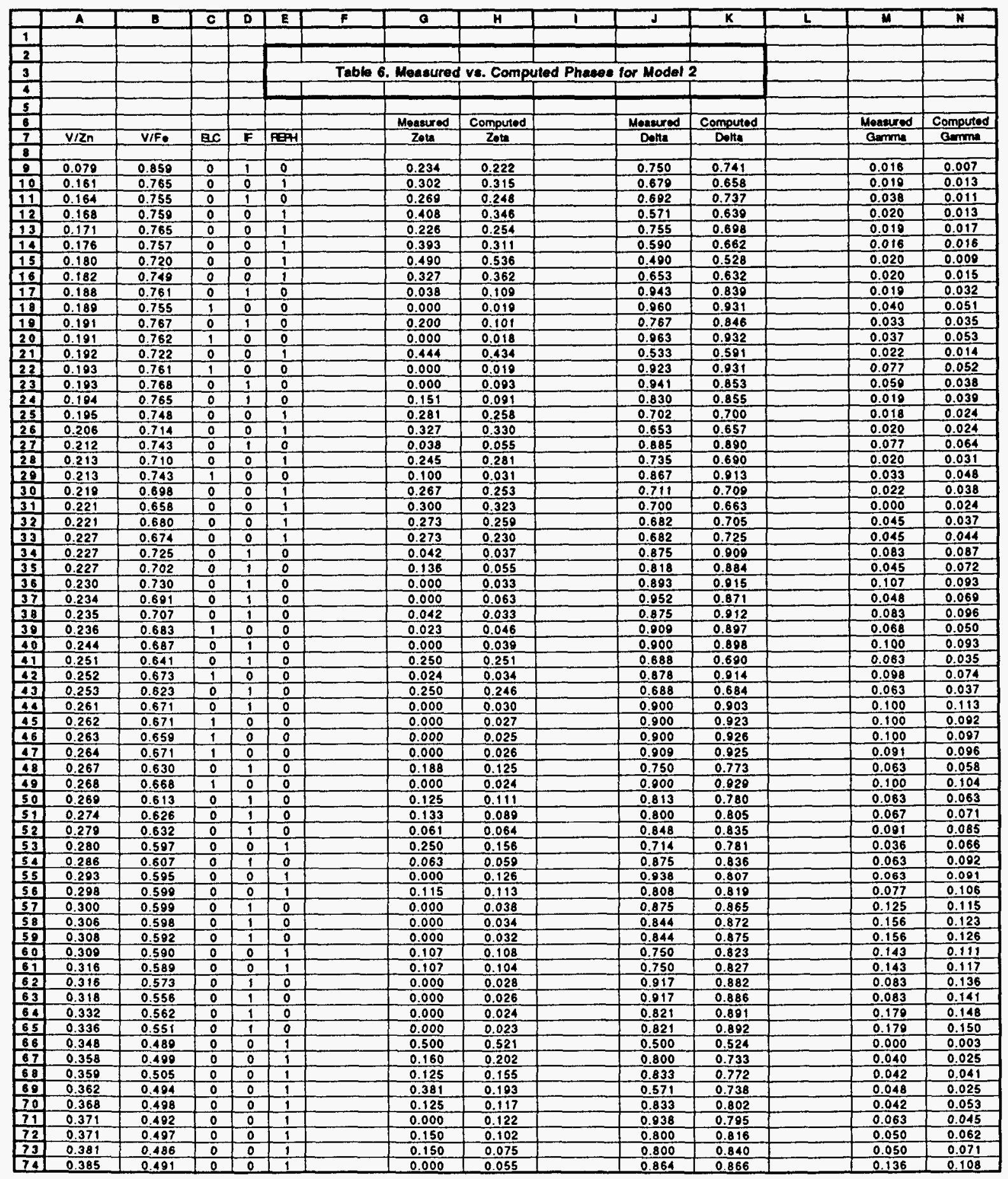


Model 2, precisely the same information that Tables 2 and 3 present for Math Model 1: the neural network numerics and the comparison between measured and computed phased.

For Math Model 1, Figures 9, 10, and 11 present scatter plots of the measured and predicted valued for the zeta, delta, and gamma layers, respectively. Clearly, the agreement is good.

\subsection{Validation of the Augmented Gauge}

The data for a completely independent test and evaluation of the DMC approach were obtained at the Stelco Hilton Works' Z-Line, where a DMC800 Zinc/Iron Coating Gauge was already installed and operational. In three days of on-line testing, the two gauge-measured signals (i.e., the relative zinc and iron voltages) for each of the galvanneal's top and bottom surfaces were recorded online at $1 \mathrm{~Hz}$, along with the corresponding phase distributions estimated by Math Models 1 and 2 running on a PC installed by DMC for this test sequence. Figure 12 illustrates the overall on-line data collection process. In addition, some twenty-six samples from the Stelco coils produced during these three days were selected for off-line examination. For these twenty-six samples, DMC made and recorded the same measurements in the same laboratory version of the on-line gauge as used for the Inland-produced samples. For these twenty-six samples, Stelco made the phase distribution measurements by using its own prescribed metallographic techniques, which differed from JPL's. Application of the unmodified Math Models 1 and 2 to these twenty-six samples yielded phase distribution estimates in substantial disagreement with the Stelco-measured values. Figures 13, 14, and 15 present the scatter plots of the measured and predicted values for the zeta, delta, and gamma phases, respectively. This result was not unanticipated, in view of recognized manufacturer-to-manufacturer differences in galvanneal production and metallography techniques from one steelmaker to another. Figure 16 displays the regions mapped out in relative-voltage space by the Inland-produced and the Stelco-produced samples.

Figures 17,18 , and 19 demonstrate that numerics fit exclusively to the twenty-six-sample Stelco training set predict the measured phases very accurately. A more meaningful extension of the DMC approach showed that manufacturer-

\footnotetext{
${ }^{4}$ The content of this subsection is drawn heavily from Reference 6.
} 


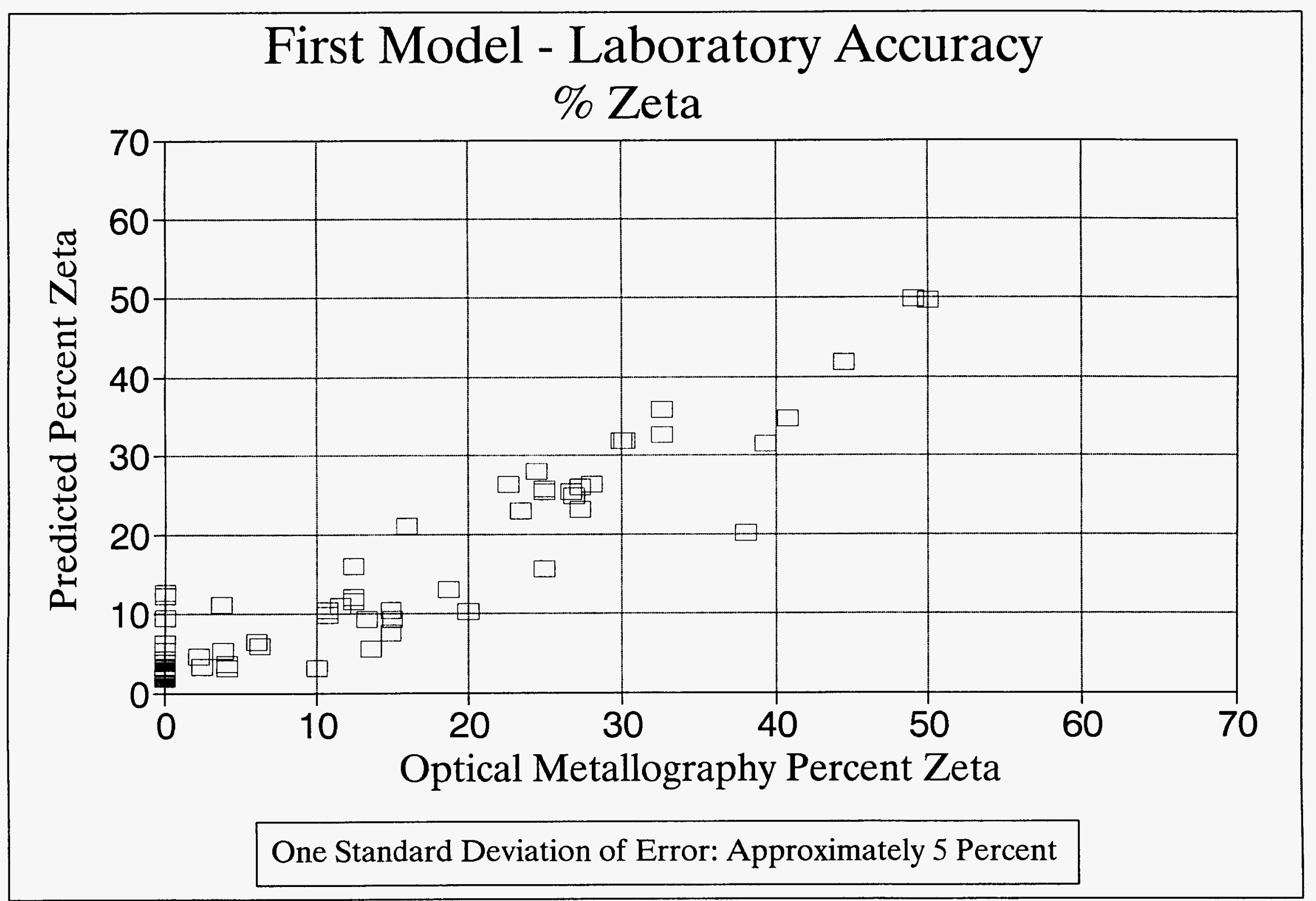




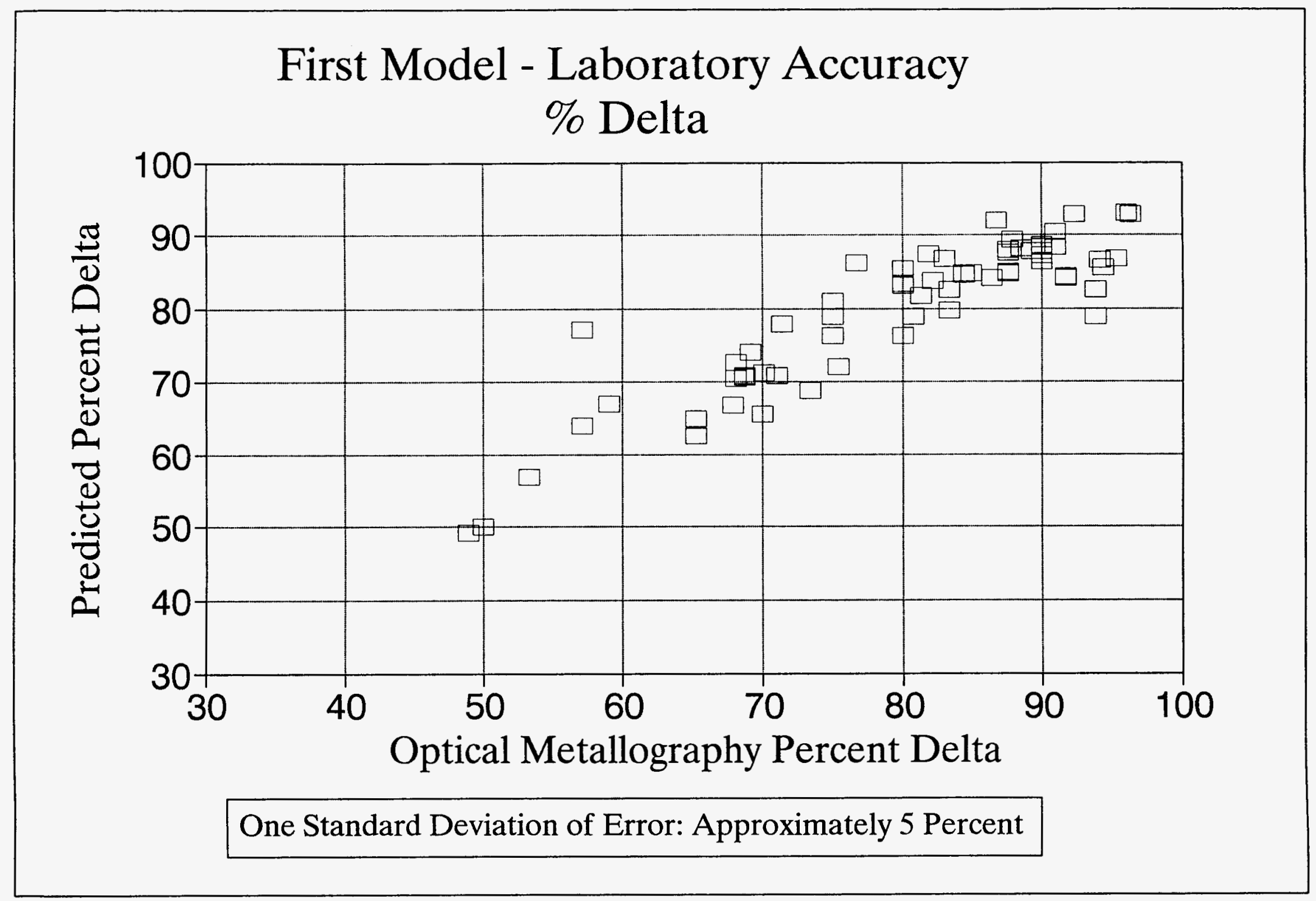




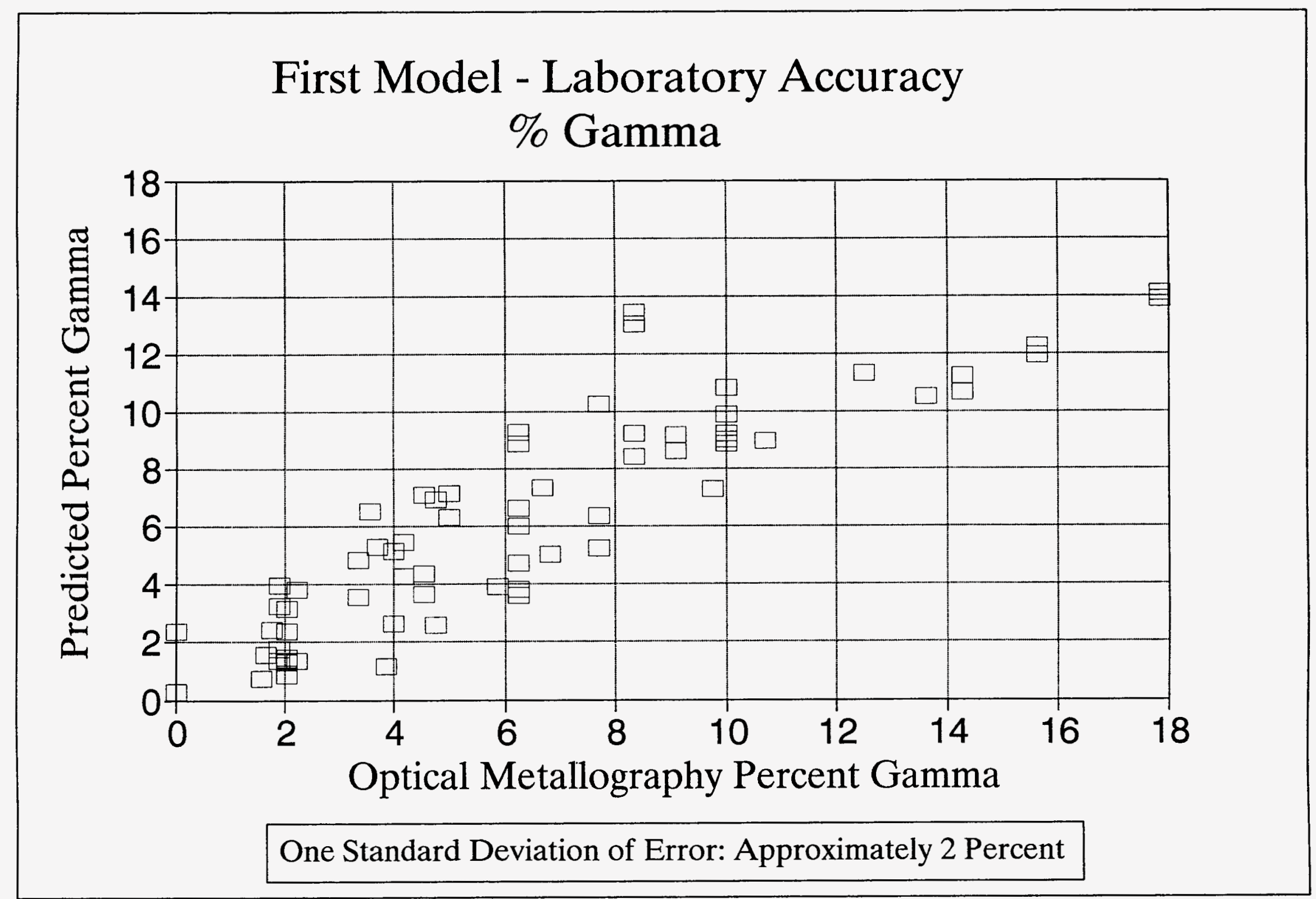


Uี

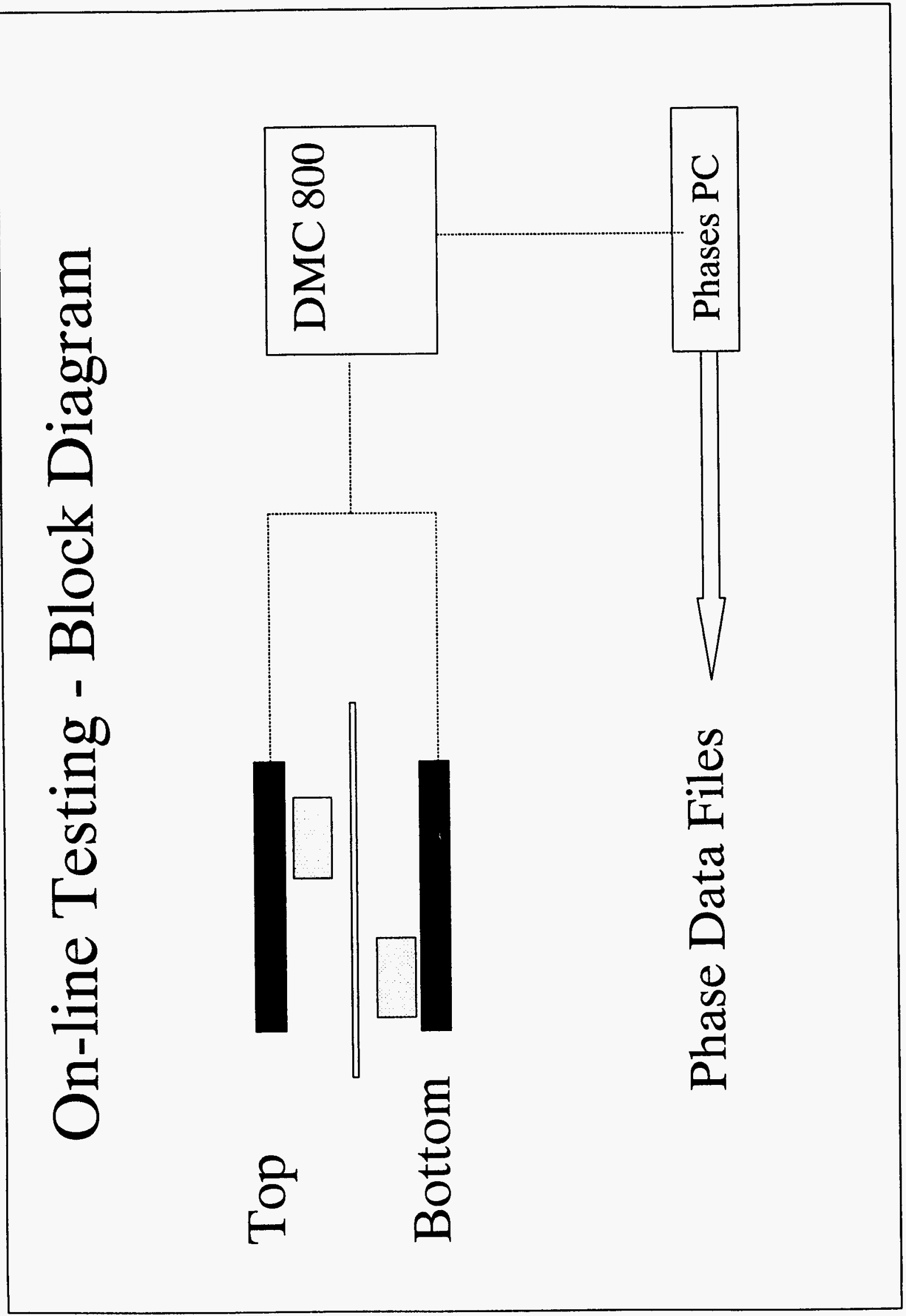

告

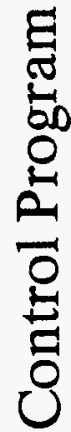

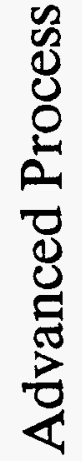

$\frac{1}{4}$ 


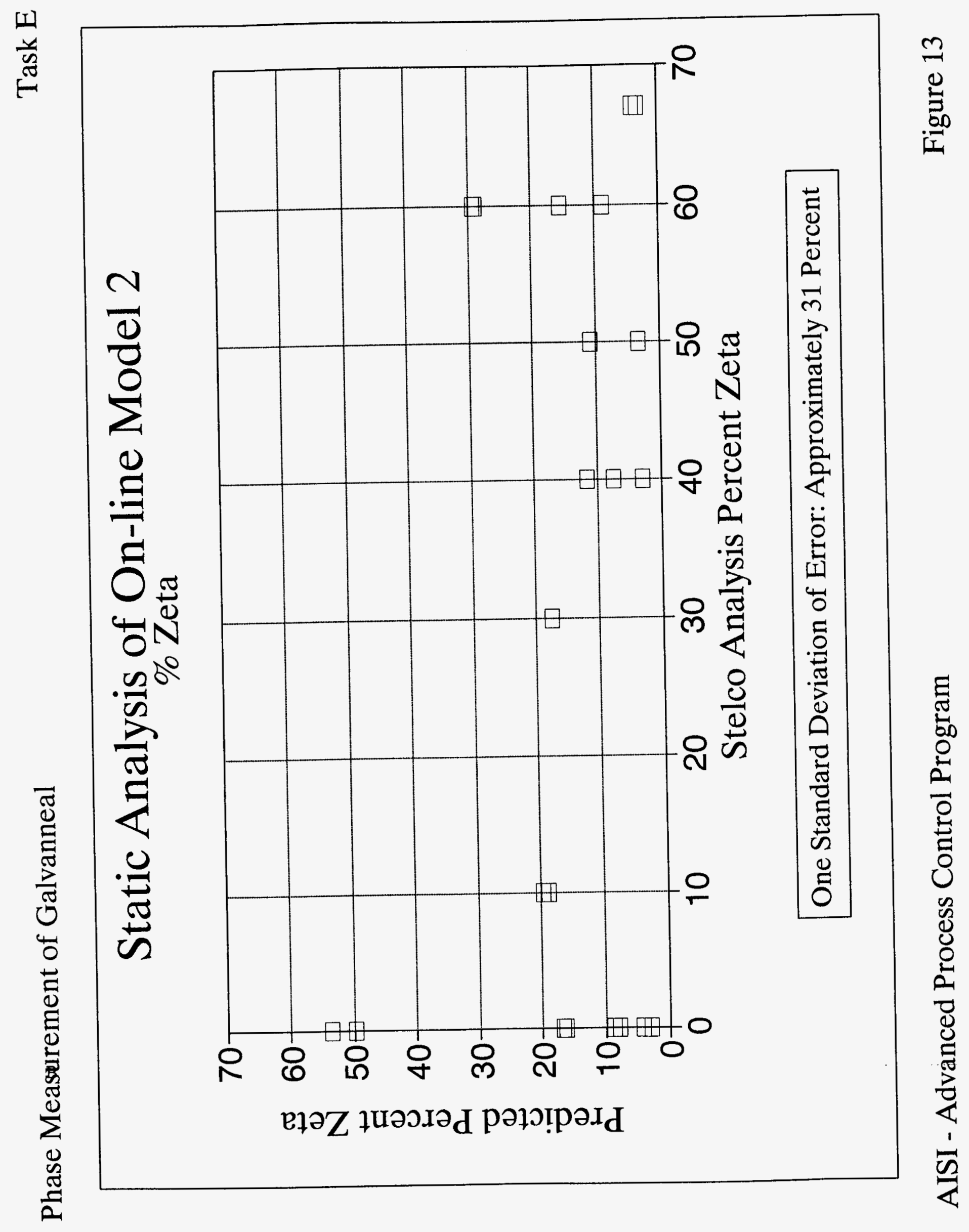




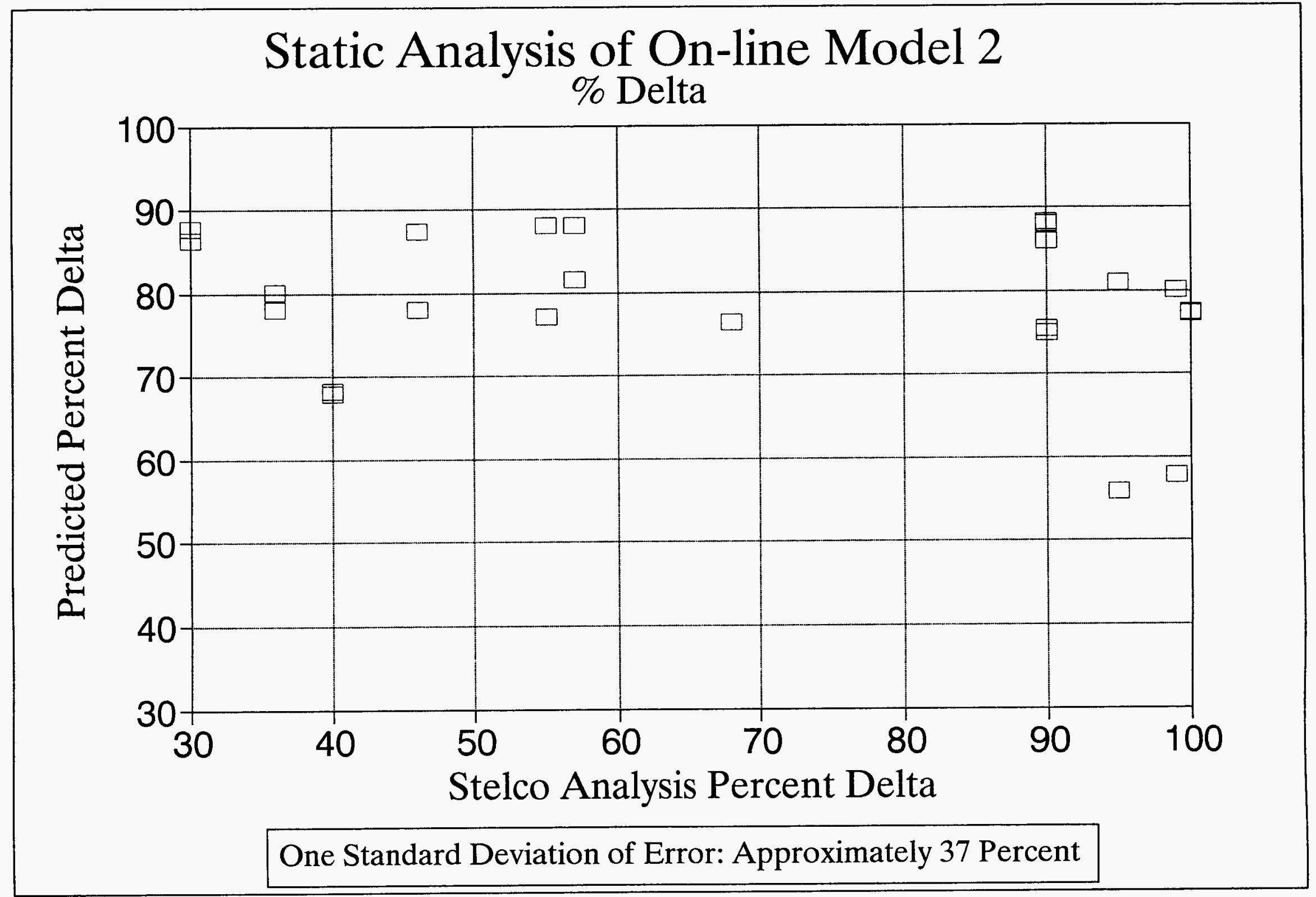




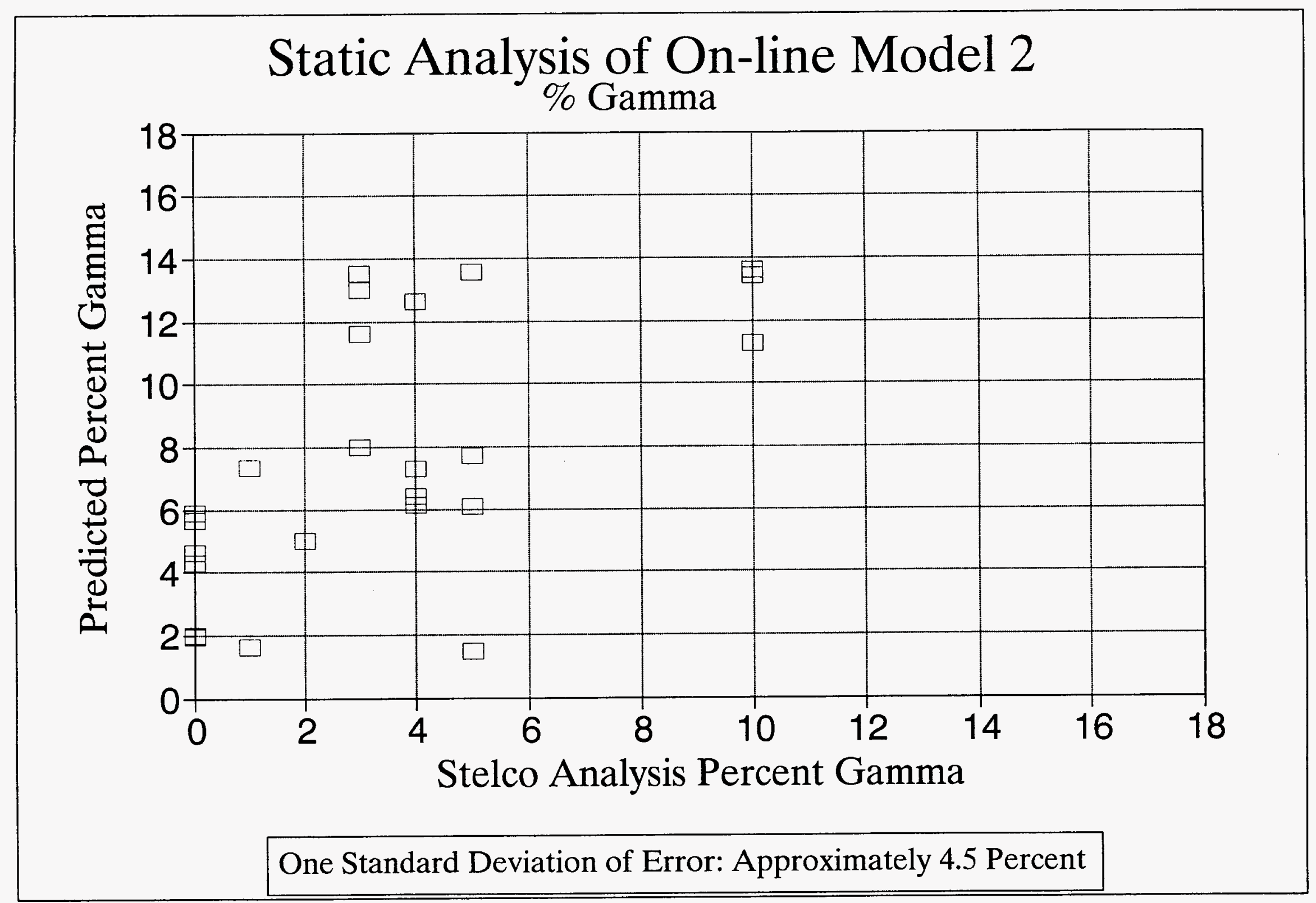




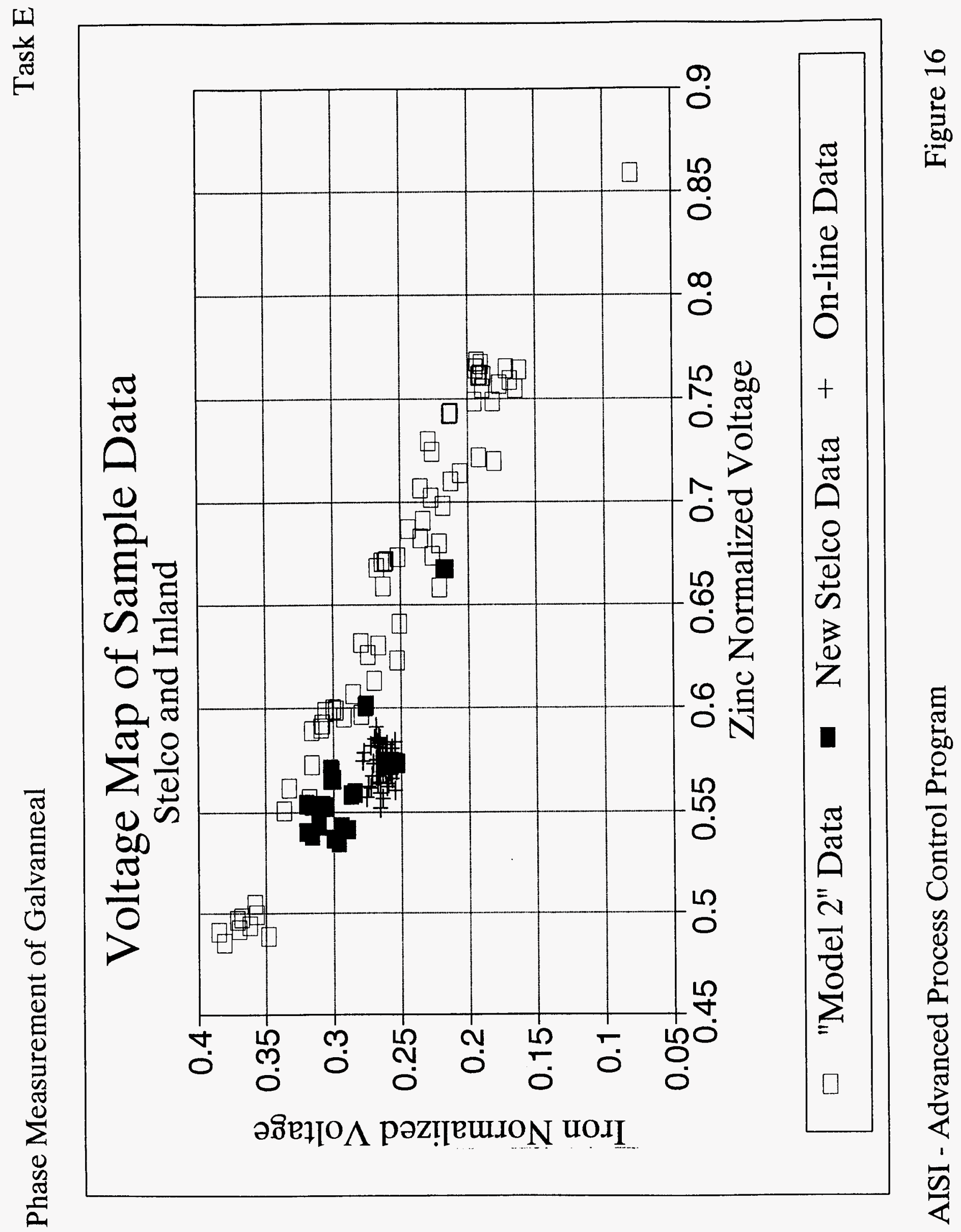




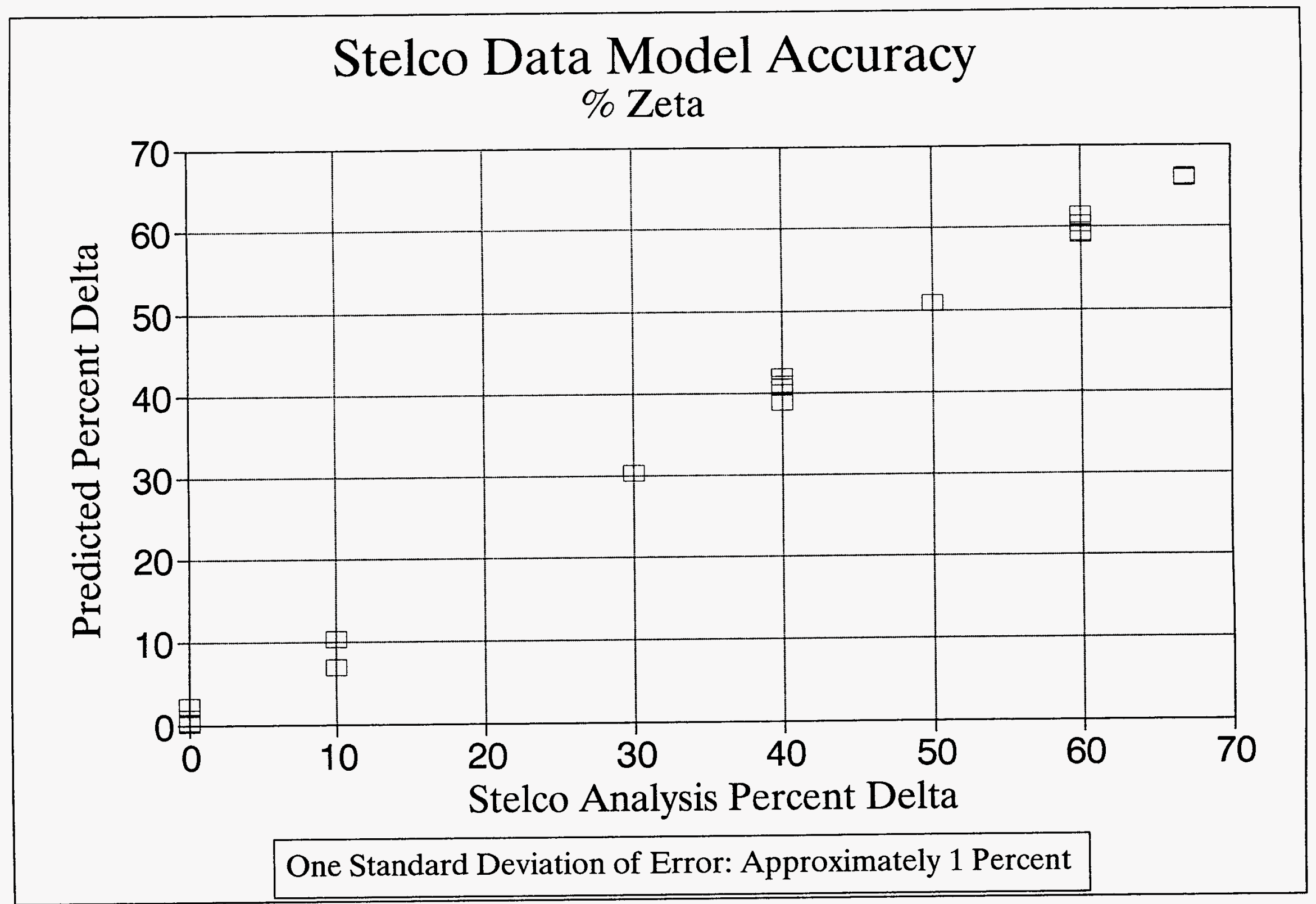




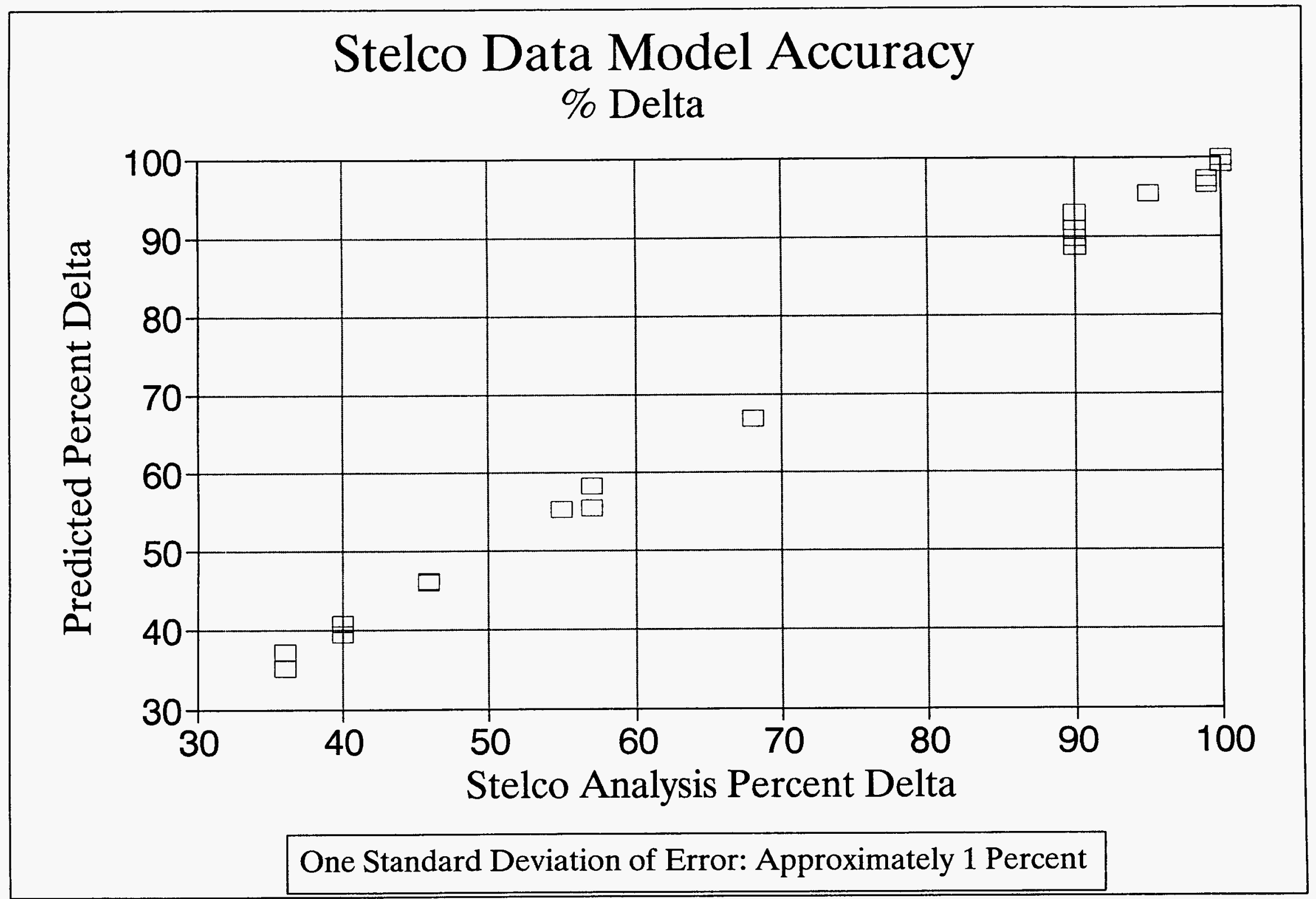




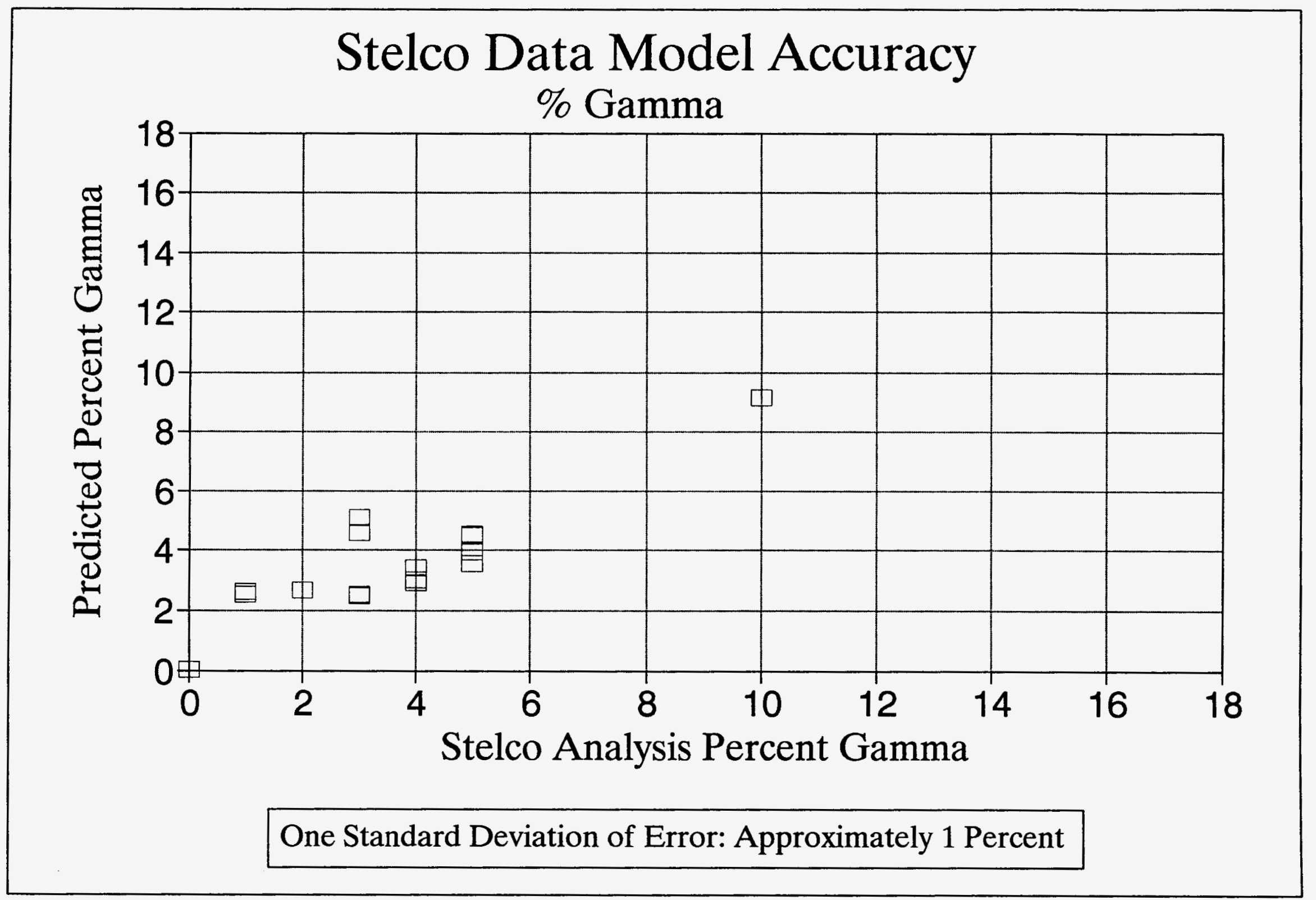


to-manufacturer disparities could be circumvented. The specific extension was predicated on an enlarged training-set consisting of the previous Math Model 2 (Inland-produced and JPL-metallographed) samples plus the newer twenty-six (Stelco-produced and Stelco-metallographed) samples; moreover, an additional input property identified the sample as having been produced by Inland or Stelco. The resulting numerical embodiment of DMC's mathematical representation is called the Composite Math Model and provides phase distribution estimates which agree well with the independently measured phase distribution for all samples for which such measurements are available.

Table 7 summarizes the Composite Math Model's training-set, consisting of ninety-two samples. In Table 7, which follows the same nomenclature conventions introduced above, the term "STELCO" designates the sixth input variable, which has value one for Stelco-produced samples and has value zero otherwise. The Composite Math Model's twenty-six samples added to Model 2's sixty-six samples are identifiable as those samples with the entry " 1 " in the column headed "STELCO." Table 8 summarizes the numerical values corresponding to the $\mathrm{Q}=53$ parameters of the Composite Math Model's neural network. Table 9 compares the measured phases with the phases calculated by the specific neural network corresponding to Table 8's numerics.

For the Composite Math Model, Figures 20, 21, and 22 compare the measured and predicted values for the zeta, delta, and gamma phases, respectively, and illustrate the agreement apparent from Table 9. These results serve not only to validate DMC's basic approach, but also to suggest precautions to be observed in porting the augmented DMC gauge from one setting to another. 


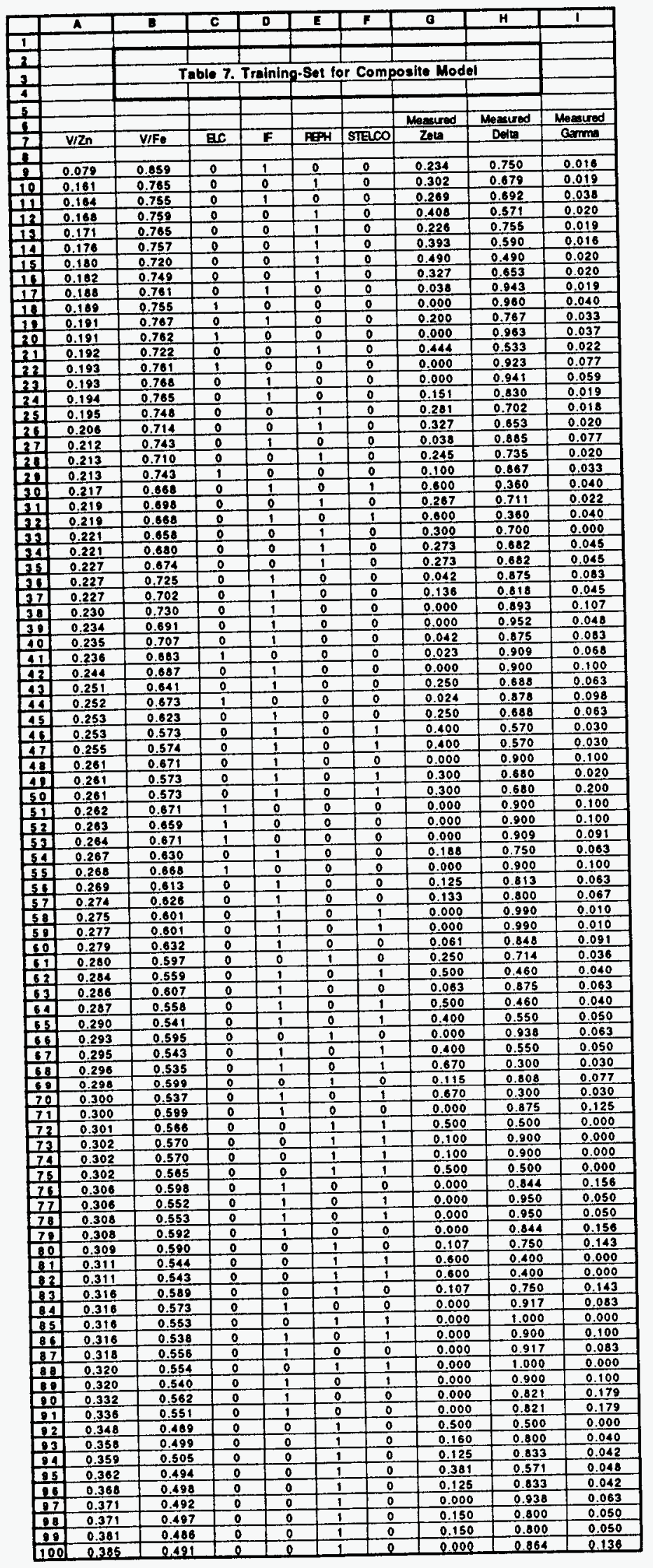


Table 8. Neural Network Coupling- and Bias-Coefficient Arrays for the Composite Model

\section{From Input Layer to Hidden Layer:}

\begin{tabular}{|c|c|c|c|c|c|}
\cline { 2 - 6 } \multicolumn{1}{c|}{} & \multicolumn{5}{c|}{ Hidden Layer } \\
\hline Input Layer & 1 & 2 & 3 & 4 & 5 \\
\hline 1 & 22.336571 & 21.940987 & -47.369106 & 15.421634 & -115.815903 \\
\hline 2 & -10.367128 & 5.739759 & 10.084509 & -40.163631 & -68.238846 \\
\hline 3 & -2.19391 & -5.660607 & -9.42646 & -2.077162 & 1.327517 \\
\hline 4 & 0.648467 & -1.044263 & 0.772363 & 10.361391 & 15.933495 \\
\hline 5 & -1.590352 & -2.128625 & 8.46094 & 1.602931 & 15.835445 \\
\hline 6 & -10.92958 & 9.110885 & -2.103897 & -11.320119 & 32.361069 \\
\hline
\end{tabular}

\begin{tabular}{|c|c|c|c|c|c|}
\cline { 2 - 6 } \multicolumn{1}{c|}{} & \multicolumn{5}{c|}{ Hidden Layer } \\
\cline { 2 - 6 } \multicolumn{1}{c|}{} & 1 & 2 & 3 & 4 & 5 \\
\hline Bias & 7.798959 & -5.531787 & 4.066257 & 13.034744 & 26.346346 \\
\hline
\end{tabular}

\section{From Hidden Layer to Output Layer:}

\begin{tabular}{|c|c|c|c|}
\cline { 2 - 4 } \multicolumn{1}{c|}{} & \multicolumn{3}{c|}{ Output Layer } \\
\hline $\begin{array}{c}\text { Hidden } \\
\text { Layer }\end{array}$ & 1 & 2 & 3 \\
\hline 1 & 12.487509 & -9.65997 & 5.384381 \\
\hline 2 & -8.945512 & 6.952096 & 4.755915 \\
\hline 3 & 7.874952 & -5.747142 & -4.1611 \\
\hline 4 & 7.462153 & -5.779438 & -3.609717 \\
\hline 5 & 21.696442 & -16.650782 & -0.216048 \\
\hline
\end{tabular}

\begin{tabular}{|c|c|c|c|}
\cline { 2 - 4 } \multicolumn{1}{c|}{} & \multicolumn{3}{c|}{ Output Layer } \\
\cline { 2 - 4 } & 1 & 2 & 3 \\
\hline Bias & -14.299695 & 10.738599 & -8.343033 \\
\hline
\end{tabular}




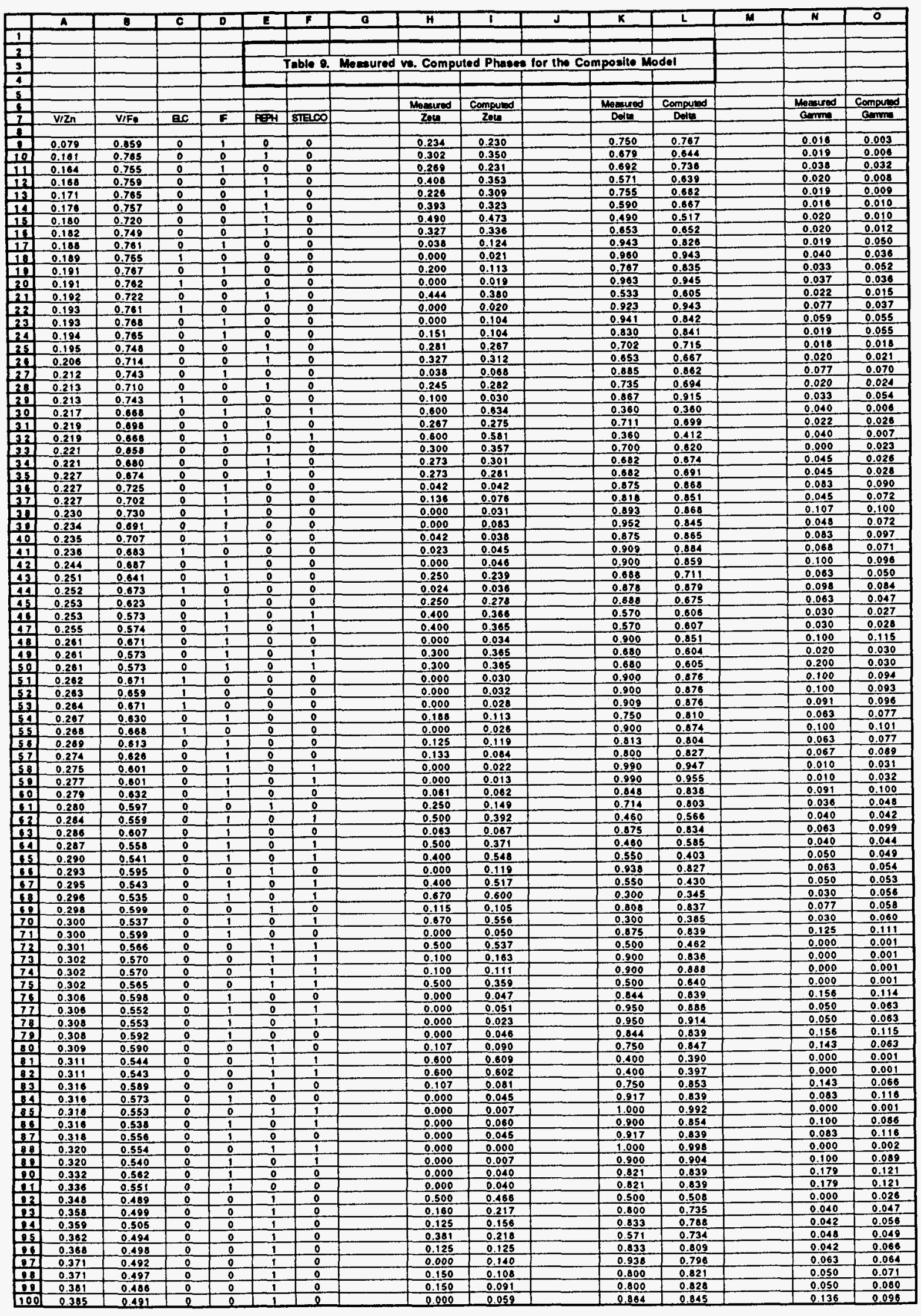


प1

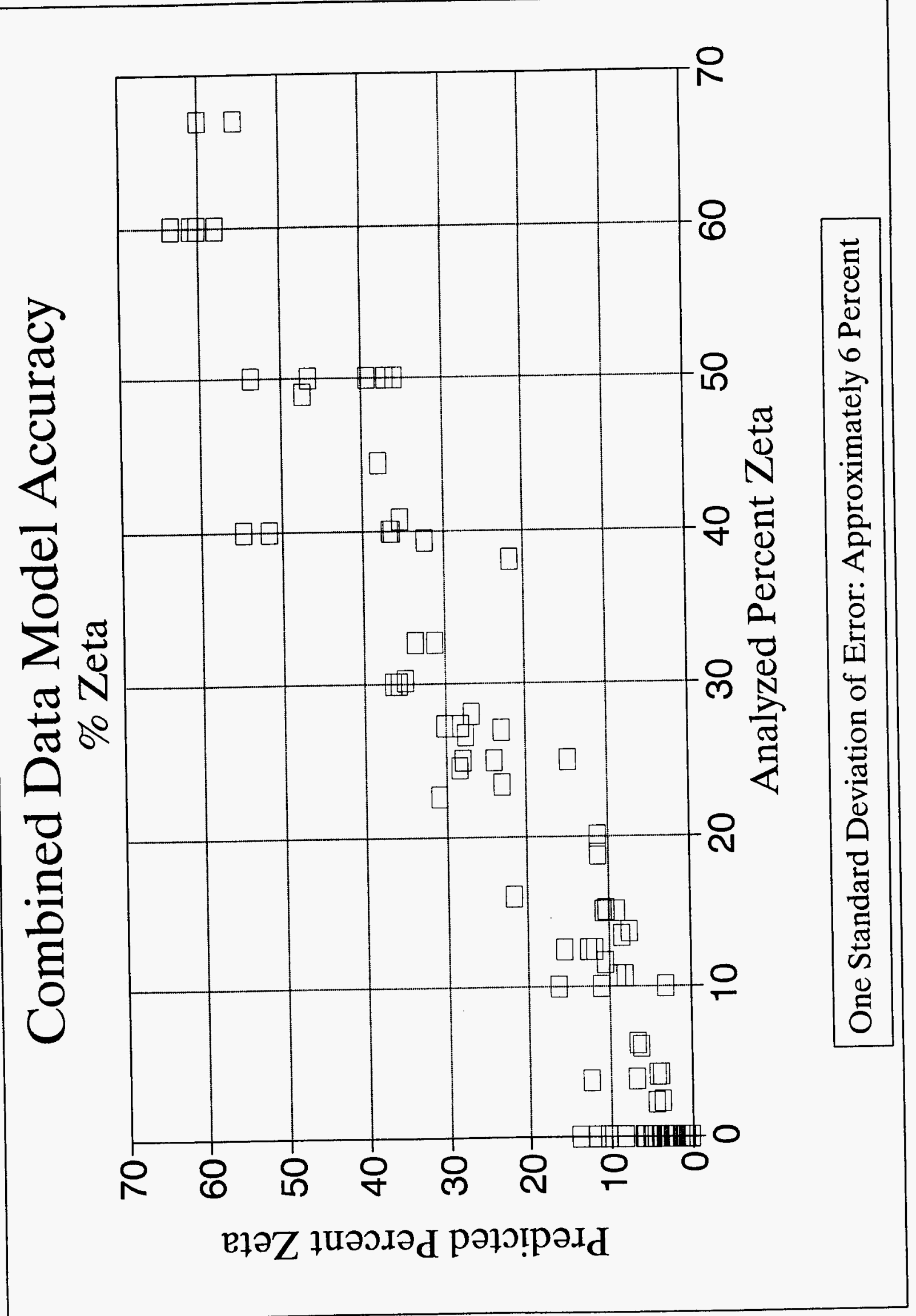

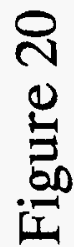

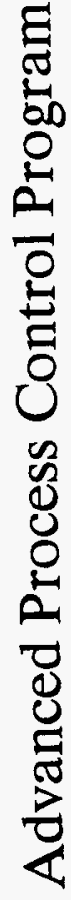

$\frac{1}{2}$ 
2
0
0
0
0
0
0
0
0
0
8
0
0
0
0
0
0
0
0
0
0
0
0
0
0

Predicted Percent Delta

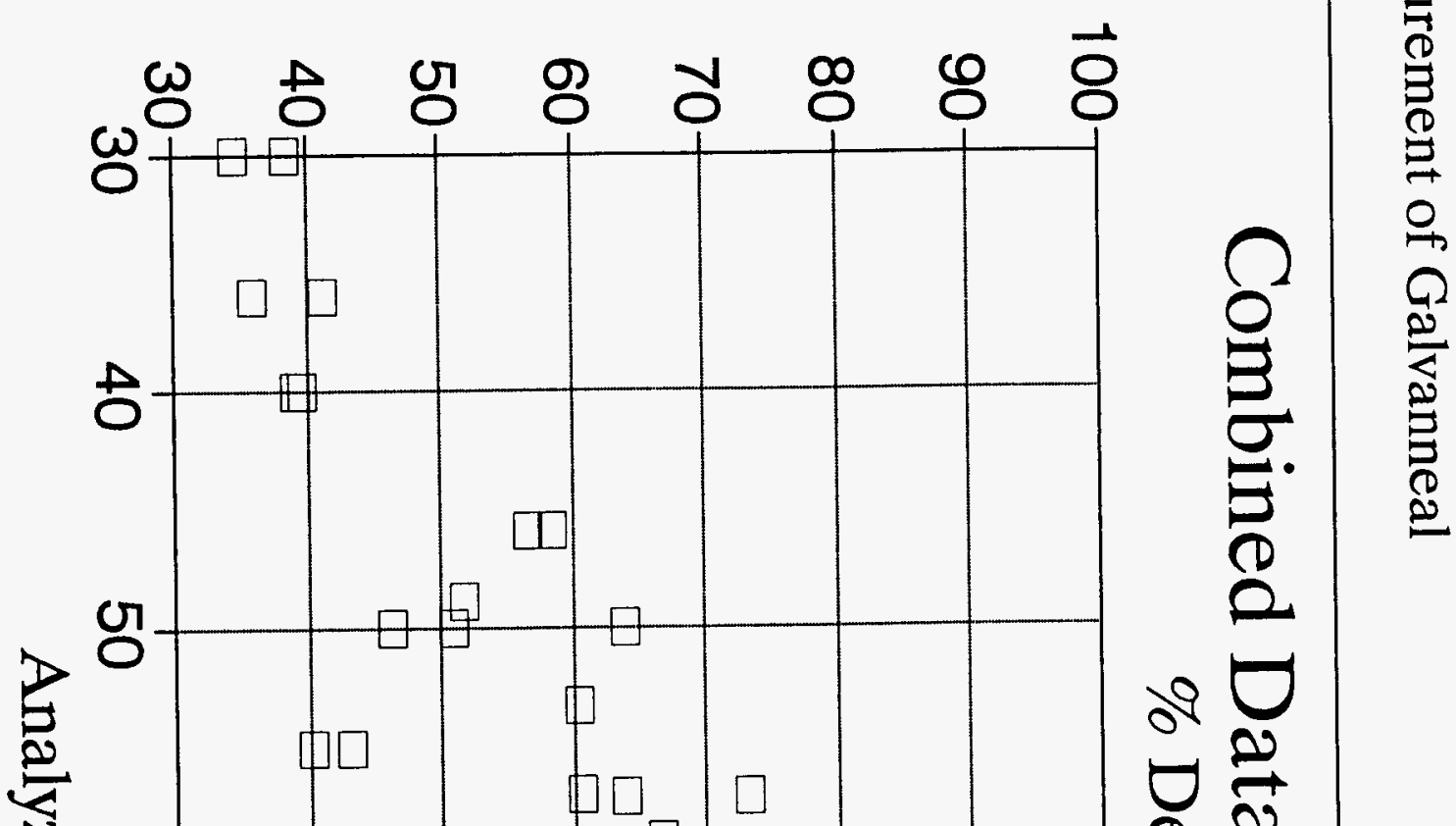

N. 몸

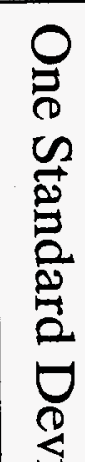

D.

官.

P

꿍

(D)

8

$\frac{1}{8}$

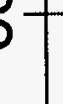

$\frac{8}{2}$

$D$

V

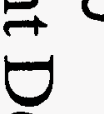

ㄹ.

常

$\frac{5}{2}$

a

7

$\frac{1}{8}$

艿

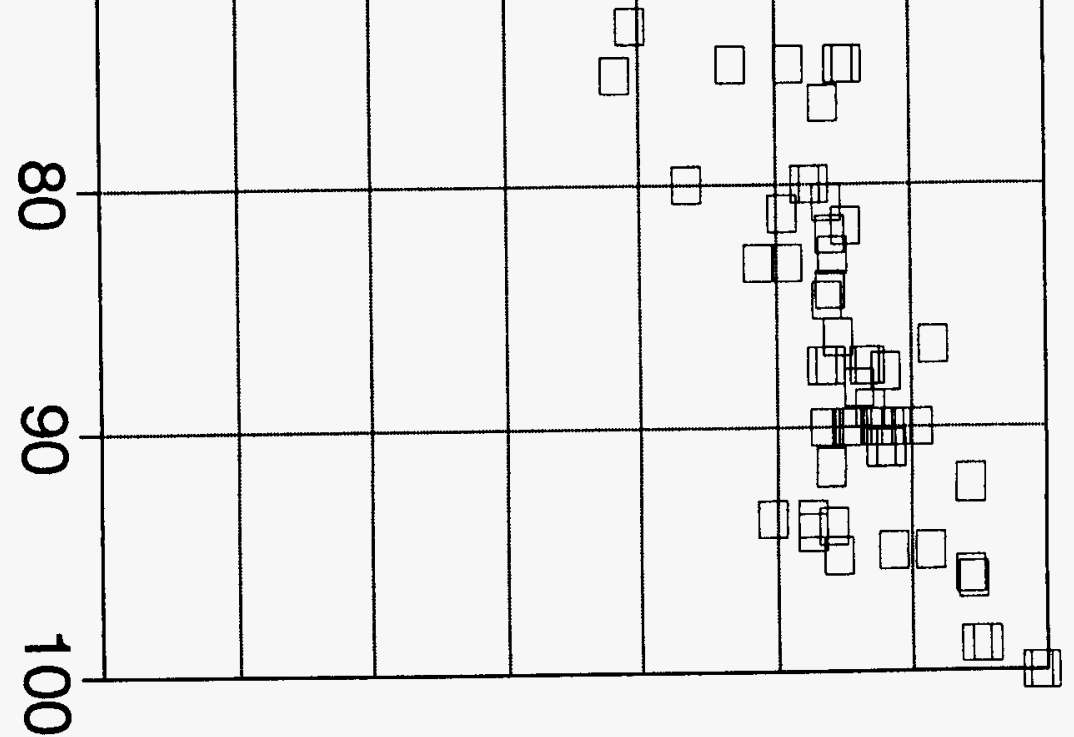




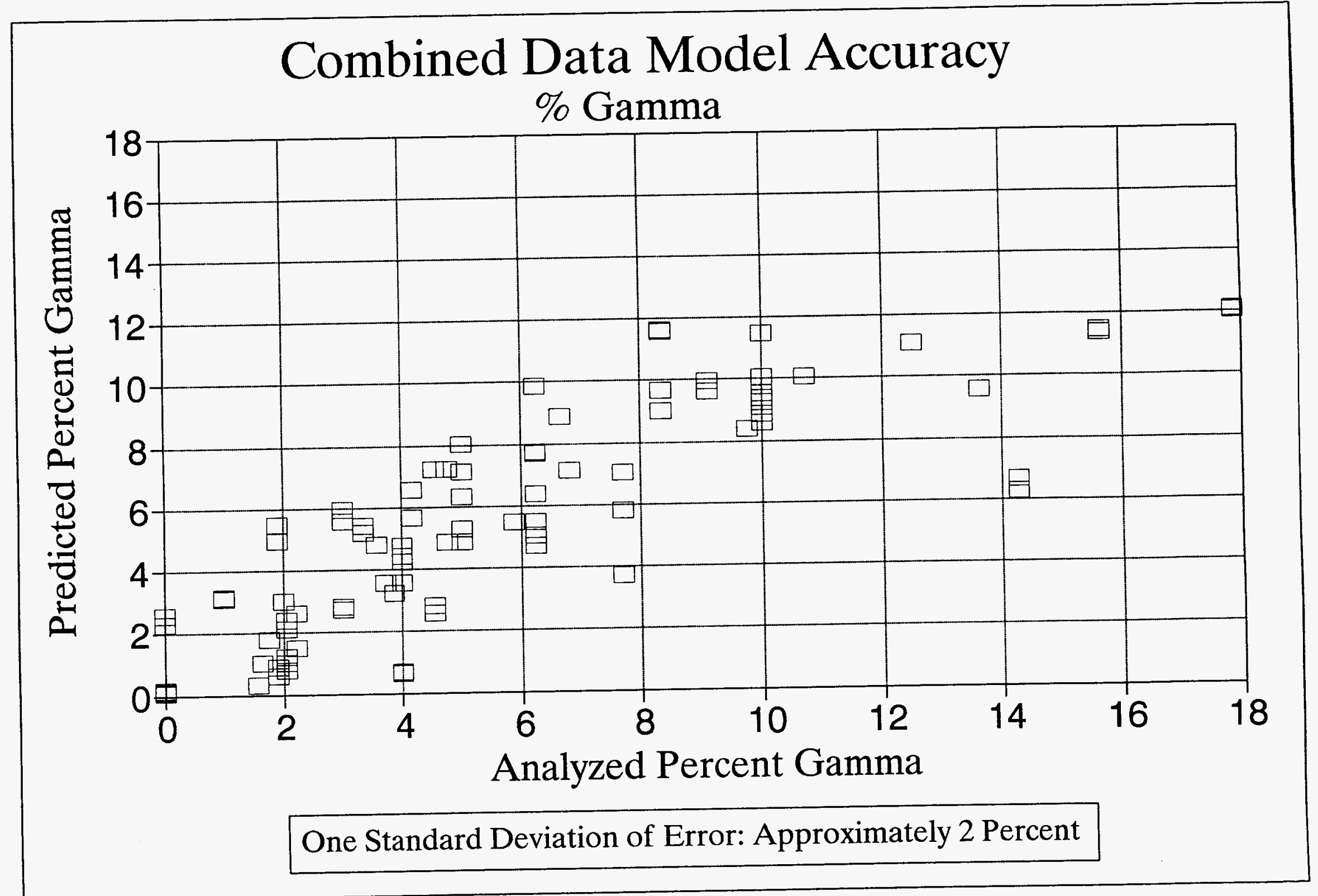




\section{CONCLUSIONS AND RECOMMENDATIONS}

This report highlights the results achieved by Data Measurement Corporation as one participant in one project of the six projects constituting the DOE/AISI Advanced Process Control Program. As directed and monitored by the sponsors, each project's management approach entailed joint and active participation and "partnering" by organizations representing steelmakers, advanced technology companies, and research institutions. Likewise, each project's technical approach was intended to emerge from vigorous intra-project collaboration and technical interchanges among that project's participants and to receive timely communication throughout the program. Ultimately, of course, each project was expected to produce technical results for comparison with the project's objective.

DMC's experience with Project E, aimed at developing an industrial gauge for measuring the phase distribution of galvanneal, has demonstrated that within this project,

- the management approach's partnering alignment exhibited powerful synergy and efficiency,

- the intra-project collaboration produced a technical approach that was clear, comprehensive, and flexible,

- following the project's technical approach produced results that realize the project's goal, viz., the development of an on-line instrument for continuous reading of the zinc-iron phase distribution in the galvanneal coating, using $\mathrm{x}$-ray fluorescence and software analytical techniques,

- flexibility and innovation were needed and demonstrated in adapting the project's approach to a galvanneal-production environment not previously reflected in the training-set.

For these accomplishments to remain vital, DMC suggests that

- the lessons learned during this project be recognized, applied, and extended, 
- further steelmaker-DMC research collaboration seek to define, with flexible realism, the strategy for creating manufacturer-specific samples to enlarge the training-set. 


\section{REFERENCES}

1 American Iron and Steel Institute, Advanced Process Control Research Program for the Steel Industry, Proposal to the U.S. Department of Energy, March 2, 1992.

2 Minutes of the Project E First Year DOE Review Meeting, March 10, 1994.

3 Data Measurement Corporation, High Speed Zinc/Iron Coating Gauge, July 1992 Brochure.

4 Jacques J. Vidal, Exploring Neural Networks with Mathematica, UCLA Course Notes, December 1, 1992, Mathematica Notebook Version Available as MathSource item number 0204-523, Wolfram Research, Inc.

5 James A. Freeman, Simulating Neural Networks with Mathematica, Addison-Wesley, 1993, ISBN: 0-201-56629-X.

6 Minutes of the Project E Second Year DOE Review Meeting, May 20, 1995. 